\title{
A Multiphase Timing-Skew Calibration Technique Using Zero-Crossing Detection
}

\author{
Chung-Yi Wang, Student Member, IEEE, and Jieh-Tsorng Wu, Senior Member, IEEE
}

\begin{abstract}
This paper describes a timing-skew calibration technique which equalizes the phase spacings among multiphase clocks. The scheme uses simple sample-and-hold circuits controlled by the multiphase clocks to sample a common reference input. Phase spacing is measured by counting the number of zero crossings between two adjacent sampling sequences. A zero-crossing detection scheme is proposed. It has better immunity against the offsets of the comparators used in the detector. A digital calibration processor is also proposed. It examines the outputs from the zero-crossing detectors, and then adjusts the delays of clock buffers in order to minimize timing skews. The proposed calibration scheme does not demand stringent requirement for the reference input. Its application to a eight-channel 6-b time-interleaved analog-to-digital converter is demonstrated.
\end{abstract}

Index Terms-Analog-digital conversion, calibration, clocks, phase estimation, time-interleaved, timing circuits, timing skew.

\section{INTRODUCTION}

A MULTIPHASE clock generator generates a set of clocks that have identical frequency but different phases. Many applications, such as time-interleaved analog-to-digital converters (TI ADCs), require the clock phases to be uniformly spread over one clock period. Fig. 1 shows an $M$-channel sampling system including a multiphase clock generator. The M-phase clocks are generated using a delay line consisting of $M$ cascaded delay units with equal delay $U_{j}$, where $1 \leq j \leq M$. The delay of $U_{j}$ is usually controlled by a phase-locked loop or a delay-locked loop. For each $j$, the $B_{j}$ buffer is connected to the output of $U_{j}$ and generates the $\phi_{j}$ clock that drives the $\mathrm{SAH}_{j}$ sample-and-hold circuit. For a TI $\mathrm{ADC}$, the time interval between two consecutive samplings must remain constant. Any timing skew in the multiphase sampling clocks can yield sampling interval variation and degrade the overall signal-to-distortion-plus-noise ratio (SNDR) performance of the ADC. The timing skews are caused by the device mismatches in the delay units and the clock buffers, as well as the mismatches among clock signal routes. For an eight-channel 6-b 16-GS/s TI ADC, the skew must be less than 0.31 ps so that the ADC can attain 37-dB SNDR. This skew

Manuscript received June 18, 2008. First published October 31, 2008; current version published June 19,2009. This work was supported by the National Science Council of Taiwan, R.O.C., under Contract NSC-96-2221-E-009-177 and the MediaTek Research Center at National Chiao-Tung University. This paper was recommended by Associate Editor S. Pavan.

The authors are with the Department of Electronics Engineering, National Chiao-Tung University, Hsin-Chu 300, Taiwan (e-mail: jtwu@mail.nctu.edu. tw).

Digital Object Identifier 10.1109/TCSI.2008.2008477

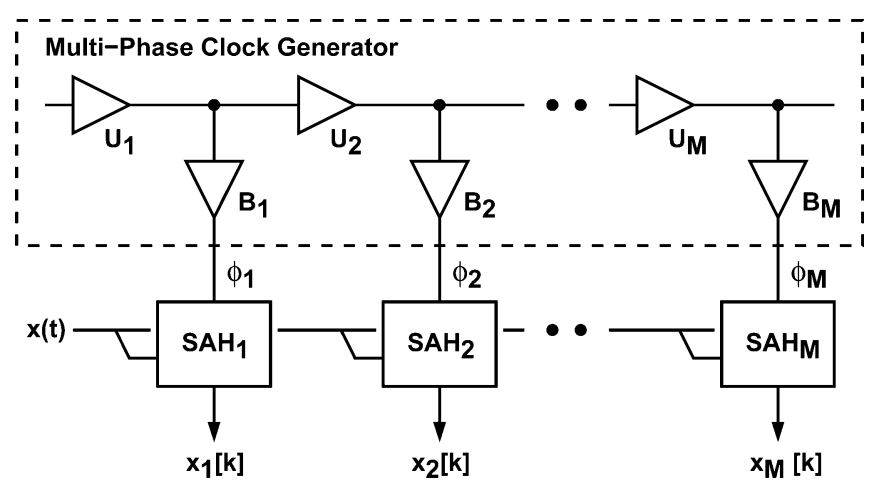

Fig. 1. Multiphase sampling system.

requirement is difficult to achieve even for chips fabricated by today's advanced integrated-circuit technologies.

There are calibration techniques to correct the timing skews. A useful calibration scheme must be able to detect the timing skew first and then make the necessary correction. Most timingskew detection techniques acquire the timing information from a reference signal. For TI ADCs, if the reference is narrowband so that it does not cause aliasing in each analog-to-digital (A/D) channel, digital signal processing algorithms, such as Fourier transform [1] or cross correlation [2], [3], can be applied for skew detection. If the reference is well defined and rich in timing information, such as a ramp signal, the skew can be detected without complex signal processing [4], [5]. However, a high-speed high-precision reference is difficult to generate. All the above skew detection techniques need to use the multibit A/D channels in the TI ADC to digitize the reference. Thus, it is not trivial to perform the calibration in the background without interrupting the normal A/D operation. To enable background calibration, the scheme of [4] adds a ramp reference to the ADC's input. This precise signal summation is difficult to implement and degrades signal dynamic range. On the other hand, the scheme of [5] requires an additional A/D channel and employs complex channel switching.

The timing skew can also be detected by counting the rising (or falling) edges of an asynchronous reference [6]-[8]. The scheme needs only simple hardware, such as flip-flops and adders. Its fundamental principle was expanded as zero-crossing detection [9]. Although stringent specifications for the references are not required, these techniques are sensitive to the input-referred offsets of the comparators used in the zero-crossing detectors. In some schemes, flip-flops serve as the comparators.

There are calibration schemes for TI ADCs that directly use the input of the ADC as the reference for timing-skew detection 


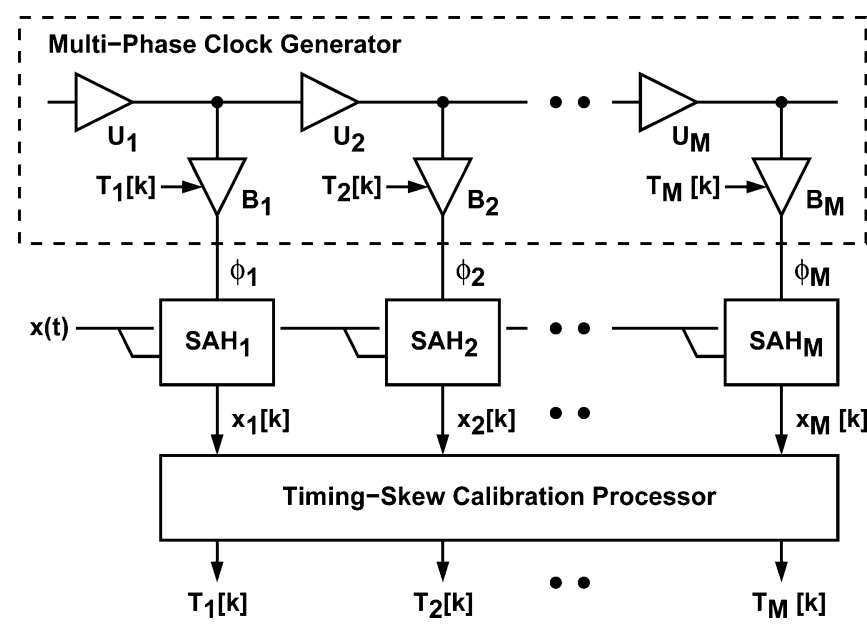

Fig. 2. Proposed architecture for multiphase timing-skew calibration.

[2], [9]. Those schemes inherently operate in the background. The scheme of [2] restricts the input to be narrowband so that it cannot cause aliasing in each $\mathrm{A} / \mathrm{D}$ channel. This narrowband restriction can be lifted by the clock-phase random-chopping technique [9]. However, the required clock choppers are difficult to implement. The mismatches among clock choppers can degrade the effectiveness of the technique. Furthermore, the effectiveness of these input-reference schemes depends on the richness of timing information residing in the inputs. For example, a dc input contains no timing information. No timing-skew calibration can function under such an input condition.

This paper describes a timing-skew calibration technique whose architecture is shown in Fig. 2. For each $j$ where $1 \leq j \leq M$, the $\mathrm{SAH}_{j}$ sample-and-hold circuit is driven by the $\phi_{j}$ clock from a multiphase clock generator. The $\mathrm{SAH}_{j}$ receives the $x(t)$ reference signal and produces the $x_{j}[k]$ sampling sequence. From the sampling sequences $x_{1}[k] \cdots x_{M}[k]$, the timing-skew calibration processor detects the timing skews between neighboring sampling channels. The $T_{j}[k]$ digital control signal from the calibration processor adjusts the delay of the $B_{j}$ clock buffer. The objective is to equalize all sampling intervals defined by the multiphase clocks. The timing-skew detection is based on the principle of zero-crossing (ZC) detection [9]. The $\mathrm{ZC}$ detection does not demand stringent specification for the $x(t)$ reference. It requires only one comparator per sampling channel. A new ZC detection scheme is proposed to reduce its sensitivity to comparators' offsets. Excluding comparators, the entire calibration processor can be realized by standard digital circuits.

An eight-channel 6-b TI ADC is used as a design example throughout this paper. It contains $M=8$ multiphase clocks. Each clock has a clock period of $T_{c}$ and a clock frequency of $f_{c}=1 / T_{c}$. Thus, the ADC's effective sampling interval is $T_{s}=$ $T_{c} / 8$ and the effective sampling rate is $f_{s}=8 f_{c}$. The ADC has an input range between \pm 1 , therefore, its magnitude resolution is defined as $V_{\mathrm{LSB}}=2 / 2^{6}=2^{-5}$. In addition, this TI ADC requires a timing resolution on the order of $T_{\mathrm{LSB}}=T_{s} / 2^{6}$.

The remainder of this paper is organized as follows. Section II introduces the concept of ZC and its mathematical background. Section III discusses the ZC detection techniques. Two ZC

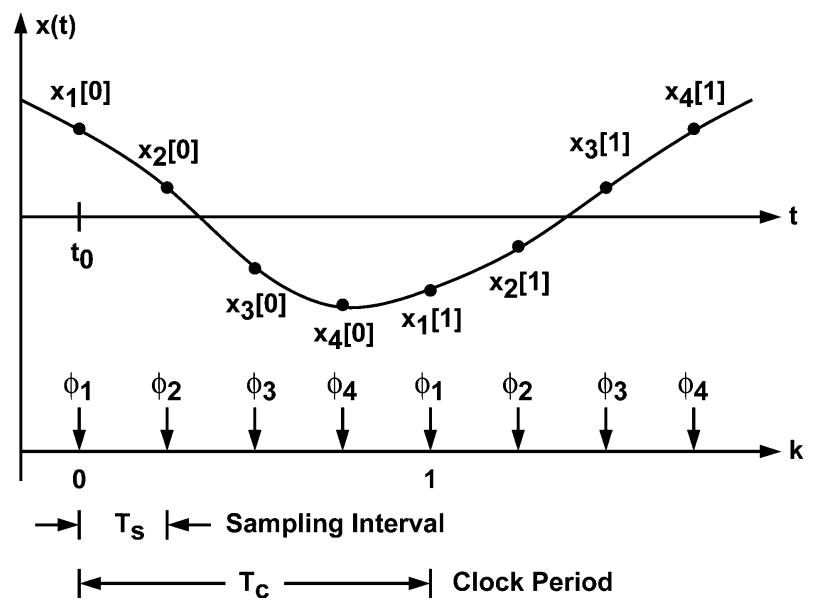

Fig. 3. $x(t)$ and $x_{j}[k]$ sampled signal.

detectors are described. Section IV describes the proposed multiphase timing-skew calibration scheme using the ZC detectors of Section III. Section V demonstrates an 8-channel 6-bit TI ADC that employs the proposed calibration scheme. Section VI draws conclusions. In addition, there are three appendices. Appendix A and Appendix B give detailed theoretical studies on the two ZC detectors described in Section III. Appendix C is a theoretical calculation of the inherent timing fluctuation of the calibration scheme described in Section IV.

\section{ZERO CROSSING}

Consider the $M$-channel sampling system shown in Fig. 1 . For each $j$ where $1 \leq j \leq M$, the $\mathrm{SAH}_{j}$ sampler triggered by the $\phi_{j}$ clock samples the $x(t)$ input and produces the $x_{j}[k]$ sampling sequence, where $k$ is a discrete time index. Both $x(t)$ and $x_{j}[k]$ for $M=4$ are illustrated in Fig. 3. If $\mathrm{SAH}_{j}$ is ideal, then $x_{j}[k]$ can be expressed as

$$
x_{j}[k]=x\left(t_{0}+(j-1) \times T_{s}+k \times M T_{s}+\tau_{j}\right) .
$$

In (1), $T_{s}$ is the nominal time interval between two consecutive samplings. The sampling rate for the entire system is $f_{s}=1 / T_{s}$. The time period of the $\phi_{j}$ clock is $T_{c}=M \times T_{s}$. Its clock frequency is $f_{c}=1 / T_{c}$. The time position of $x(t)$ relative to the multiphase clocks is represented by $t_{0}$, which is the sampling time of $x_{1}[0]$. The possible value of $t_{0}$ is between 0 and $T_{c}$. Finally, $\tau_{j}$ is the individual timing offset of the $\phi_{j}$ clock. The value of $t_{0}$ is defined in such a way that the mean of $\tau_{j}$ is zero, i.e., $\tau_{1}+\tau_{2}+\cdots+\tau_{M}=0$. A timing skew occurs between the clocks $\phi_{j}$ and $\phi_{j+1}$ if $\tau_{j} \neq \tau_{j+1}$.

Consider a $x(t)$ signal that is continuous in time and in magnitude. As time progresses, a ZC occurs if $x(t)$ changes its polarity from positive to negative or from negative to positive. For example, in Fig. 3, there is a ZC between $x_{2}[0]$ and $x_{3}[0]$, and another ZC between $x_{2}[1]$ and $x_{3}[1]$. There exists at least one ZC between $x_{j}[k]$ and $x_{j+1}[k]$ if $x_{j}[k] \times x_{j+1}[k]<0$. If $x(t)$ is a stationary Gaussian process with zero mean, then the probability of $x_{j}[k] \times x_{j+1}[k]<0$ for an arbitrary $k$ is [10], [11]

$$
P_{j, j+1}^{z}=\frac{1}{2}-\frac{1}{\pi} \sin ^{-1} \rho_{j, j+1}
$$




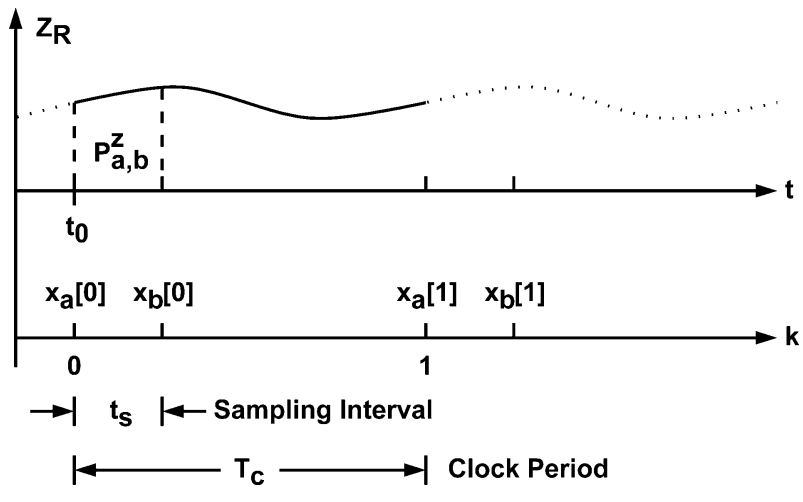

Fig. 4. ZC density.

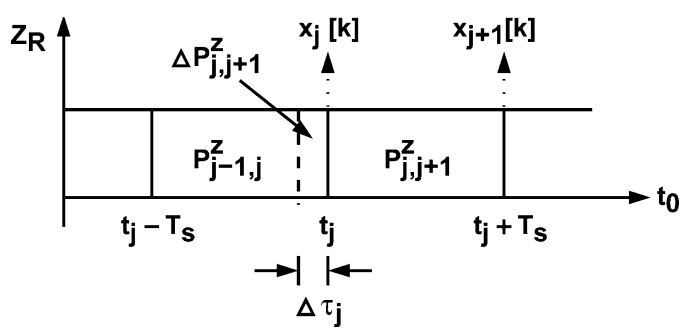

Fig. 5. Constant ZC density and timing-skew detection.

with

$$
\begin{aligned}
\rho_{j, j+1} & =\frac{E\left\{x_{j}[k] \times x_{j+1}[k]\right\}}{\sigma_{j} \times \sigma_{j+1}} \\
\sigma_{j} & =\sqrt{E\left[x_{j}^{2}\right.} \\
\sigma_{j+1} & =\sqrt{E\left[x_{j+1}^{2}\right.}
\end{aligned}
$$

where $\sigma_{j}$ and $\sigma_{j+1}$ are the standard deviations of $x_{j}[k]$ and $x_{j+1}[k]$, respectively. Furthermore, $\rho_{j, j+1}$ denotes the cross correlation between $x_{j}[k]$ and $x_{j+1}[k]$. The value of $\rho_{j, j+1}$ is between +1 and -1 . If $x(t)$ is a dc signal, then both $x_{j}[k]$ and $x_{j+1}[k]$ are constant, $x_{j}[k]=x_{j+1}[k], \rho_{j, j+1}=1$, and $P_{j, j+1}^{z}=0$. Therefore, there is no ZC for a dc $x(t)$. If $x(t)$ is a single-tone sinewave with frequency at $1 /\left(2 T_{s}\right)$, then both $x_{j}[k]$ and $x_{j+1}[k]$ are also constant, but $x_{j}[k]=-x_{j+1}[k]$, $\rho_{j, j+1}=-1$, and $P_{j, j+1}^{z}=1$, i.e., a ZC always occurs between $x_{j}[k]$ and $x_{j+1}[k]$ for every $k$.

Consider two sampling sequences $x_{a}[k]$ and $x_{b}[k]$, as illustrated in Fig. 4. Both sequences have an identical clock period of $T_{c}$. The sampling interval between $x_{a}[k]$ and $x_{b}[k]$ is $t_{s}$. The sampling time for $x_{a}[0]$ is $t_{0}$. For a generic $x(t)$ input, the cross correlation of (3) between $x_{a}[k]$ and $x_{b}[k]$ is denoted as $\rho_{a, b}\left(t_{0}, T_{c}, t_{s}\right)$. Note that $\rho_{a, b}\left(t_{0}, T_{c}, t_{s}\right)$ is a periodic function of $t_{0}$ and has a period of $T_{c}$. From (2), the corresponding $\mathrm{ZC}$ probability between the two sampling sequences is $P_{a, b}^{z}\left(t_{0}, T_{c}, t_{s}\right)$. The ZC density, defined as the ZC probability per unit $t_{s}$ time, can be expressed as

$$
\begin{aligned}
Z_{R}\left(t_{0}, T_{c}\right) & \equiv \lim _{t_{s} \rightarrow 0} \frac{P_{a, b}^{z}\left(t_{0}, T_{c}, t_{s}\right)}{t_{s}} \\
& =\frac{1}{\pi} \times\left.\sqrt{\frac{-\partial^{2} \rho_{a, b}\left(t_{0}, T_{c}, t_{s}\right)}{\partial t_{s}^{2}}}\right|_{t_{s}=0} .
\end{aligned}
$$

For the sampling system of Fig. 1, the ZC probability between $x_{j}[k]$ and $x_{j+1}[k]$ can be expressed as

$$
P_{j, j+1}^{z}=\int_{t_{j}}^{t_{j}+T_{s}} Z_{R}\left(t_{0}, T_{c}\right) d t_{0}
$$

where $t_{j}=t_{0}+(j-1) \times T_{s}$.

Consider $x(t)=A \sin \left(2 \pi f_{i} t\right)$. The sinewave input has a frequency of $f_{i}$ and an amplitude of $A$. The corresponding $\rho_{a, b}\left(t_{0}, T_{c}, t_{s}\right)$ is $\cos \left(2 \pi f_{i} t_{s}\right)$, and the corresponding $Z_{R}\left(t_{0}, T_{c}\right)$ is

$$
\begin{aligned}
& Z_{R}\left(t_{0}, T_{c}\right) \\
& \quad= \begin{cases}2 f_{i}, & \frac{f_{i}}{f_{c}} \neq \frac{p}{q} \\
\frac{2}{q} \sum_{n=0}^{p-1} \delta\left(t_{0}-n \times \frac{T_{c}}{p}\right), & \frac{f_{i}}{f_{c}}=\frac{p}{q} q \text { is even } \\
\frac{1}{q} \sum_{n=0}^{2 p-1} \delta\left(t_{0}-n \times \frac{T_{c}}{2 p}\right), & \frac{f_{i}}{f_{c}}=\frac{p}{q} q \text { is odd }\end{cases}
\end{aligned}
$$

where $f_{c}=1 / T_{c}$ is the clock frequency, and $p$ and $q$ are two mutually prime positive integers. If the $f_{i} / f_{c}$ ratio is irrational, i.e., $x(t)$ is asynchronous to the $f_{c}$ clock, then $Z_{R}$ is equal to $2 f_{i}$ and independent of $t_{0}$ and $T_{c}$. If $f_{i} / f_{c}=p / q$, then $x(t)$ is synchronous with the $f_{c}$ clock. In a synchronous case, ZCs occur only at certain instants if all clock periods are folded as one. There are $p$ possible uniformly spaced ZC instants if $q$ is even. On the other hand, there are $2 p$ possible uniformly spaced $\mathrm{ZC}$ instants if $q$ is odd.

The timing-skew calibration scheme proposed in this paper requires a $x(t)$ reference signal whose $Z_{R}$ is nonzero and independent of $t_{0}$. One example is an asynchronous sinewave whose $Z_{R}$ is $2 f_{i}$ as described in (7). As illustrated in Fig. 5, if $Z_{R}$ is constant, then the ZC probability between $x_{j}[k]$ and $x_{j+1}[k]$ is proportional to the $T_{s}$ sampling interval, i.e., $P_{j, j+1}^{z}=Z_{R} \times T_{s}$. If timing skew occurs and the sampling interval becomes $T_{s}+$ $\Delta \tau_{j}$, the ZC probability is deviated by an amount of $\Delta P_{j, j+1}^{z}=$ $Z_{R} \times \Delta \tau_{j}$. By comparing $P_{j, j+1}^{z}$ and $P_{j+1, j+2}^{z}$, one can determine whether the sampling interval between $x_{j}[k]$ and $x_{j+1}[k]$ is larger or smaller than the sampling interval between $x_{j+1}[k]$ and $x_{j+2}[k]$.

The $x(t)$ reference signal required by the proposed calibration scheme is not restricted to single-tone sinewaves. Any $x(t)$ signal can have a $Z_{R}$ independent of $t_{0}$ if it is narrowband and asynchronous to the sampling clocks. Referring to Fig. 4, assume both $x_{a}[k]$ and $x_{b}[k]$ are sampling sequences with $T_{c}=$ $1 / f_{c}$ sampling interval. If the bandwidth of $x(t)$ is less than $f_{c} / 2$ so that it does not cause aliasing, then both $x_{a}[k]$ and $x_{b}[k]$ observe the same signal but with different delays. As a result, $\rho_{a, b}\left(t_{0}, T_{c}, t_{s}\right)$ is only a function of the $t_{s}$ delay difference. From (5), the corresponding $Z_{R}$ is independent of $t_{0}$.

A $x(t)$ signal is said to be synchronous with the sampling clocks if it consists of only single-tone sinewaves that are synchronous with the sampling clocks. Its $Z_{R}\left(t_{0}, T_{c}\right)$ will comprise $\delta$-functions similar to those in (7). Under the synchronous $x(t)$ condition, the proposed calibration scheme can still function if the $Z_{R}\left(t_{0}, T_{c}\right)$ 's $\delta$-functions have the same magnitude and are uniformly spread over one $T_{c}$ period. The spacing between the $\delta$-functions must be smaller than the desired calibration timing resolution. 


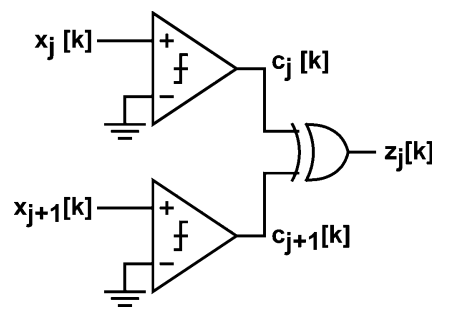

Fig. 6. Simple ZC detector (ZCD1).

\section{ZC DETECTION}

\section{A. Simple ZC Detector (ZCD1)}

Fig. 6 shows a simple ZC detector (ZCD1) to determine if a ZC occurs between $x_{j}[k]$ and $x_{j+1}[k]$. Both samples are compared with a zero reference to determine their polarities. If the $x_{j}[k]$ 's polarity is different from the $x_{j+1}[k]$ 's polarity, then the detector issues $z_{j}[k]=1$, otherwise, $z_{j}[k]=0$. The probability of $z_{j}[k]=1$ is the $P_{j, j+1}^{z}$ of (2) or (6). This ZC detection scheme is simple but sensitive to comparators' offsets. Detailed analysis is provided in Appendix A. Assume the two comparators exhibit the input-referred offsets $O_{j}$ and $O_{j+1}$ respectively, as shown in Fig. 20. The resulting $P_{j, j+1}^{z}$ is deviated from (2) by an amount of $\Delta P_{j, j+1}^{z}$, which can be approximated by

$\Delta P_{j, j+1}^{z} \approx \frac{\rho_{j, j+1}}{2 \pi \sqrt{1-\rho_{j, j+1}^{2}}}\left(\frac{O_{j}^{2}}{\sigma_{j}^{2}}+\frac{O_{j+1}^{2}}{\sigma_{j+1}^{2}}-\frac{2}{\rho_{j, j+1}} \frac{O_{j}}{\sigma_{j}} \frac{O_{j+1}}{\sigma_{j+1}}\right)$.

The $\Delta P_{j, j+1}^{z}$ variation depends not only on the normalized offsets $O_{j} / \sigma_{j}$ and $O_{j+1} / \sigma_{j+1}$, but also on the cross correlation $\rho_{j, j+1}$. For a slowly varying $x(t), \rho_{j, j+1} \approx 1, \sigma_{j} \approx \sigma_{j+1}$, then $\Delta P_{j, j+1}^{z}$ is proportional to $\left(O_{j}-O_{j+1}\right)^{2}$. The resulting $P_{j, j+1}^{z}$ probability is sensitive to the offset mismatch. For a single-tone $x(t)$ with frequency close to $1 /\left(2 T_{s}\right), \rho_{j, j+1} \approx-1, \sigma_{j} \approx \sigma_{j+1}$, then $\Delta P_{j, j+1}^{z}$ is proportional to $\left(O_{j}+O_{j+1}\right)^{2}$. The offset sensitivity is reduced when large $x(t)$ is applied, since large $x(t)$ leads to large $\sigma_{j}$ and $\sigma_{j+1}$.

When using a ZCD1 to measure the sampling interval between $x_{j}[k]$ and $x_{j+1}[k]$ of the sampling system shown in Fig. 1, the $\Delta P_{j, j+1}^{z}$ due to offsets can lead to skew measurement error $\Delta \tau_{j, o s}$. The relationship can be expressed as

$$
\Delta P_{j, j+1}^{z}=Z_{R}\left(t_{0}, T_{c}\right) \times \Delta \tau_{j, o s}
$$

where $Z_{R}\left(t_{0}, T_{c}\right)$ is the $\mathrm{ZC}$ density defined in (5). Consider the eight-channel 6-b TI ADC defined in Section I. Assume the input $x(t)=\sin \left(2 \pi f_{i} t\right)$ is a full-range single-tone sinewave asynchronous to the $f_{c}$ clock. To illustrate a low $f_{i}$ case, let $f_{i}=\left(65519 / 2^{18}\right) f_{c} \approx 0.25 f_{c}$, then we have $Z_{R}=2 f_{i} \approx 0.5 f_{c}, \rho_{j, j+1}=+0.98$, and $\sigma_{j}^{2}=1 / 2$. The resulting $\Delta \tau_{j, o s}$ versus $\left|O_{j}-O_{j+1}\right|$ is shown in Fig. 7. The solid line is obtained using (8) and (9). The black circles are time-domain simulation results. In the simulations, a sinewave with $f_{i}$ frequency is served as the $x(t)$ input. In each simulation, $P_{j, j+1}^{z}$ is obtained by calculating the ratio of the number of times $z_{j}[k]=1$ to the total number of $k$. In Fig. 7, $\Delta \tau_{j, o s}$ and

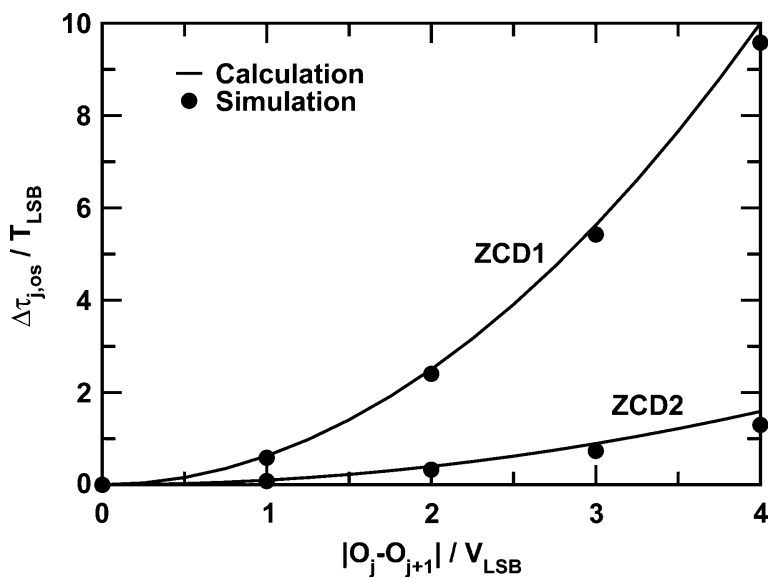

Fig. 7. Skew measurement error due to comparators' offsets. The input is a low-frequency sinewave with $f_{i} \approx 0.25 f_{c}$. The ZCD2 curve is plotted with $O_{j+1}=0$ so that $O_{j}-O_{j+1}=O_{j}+O_{j+1}$.

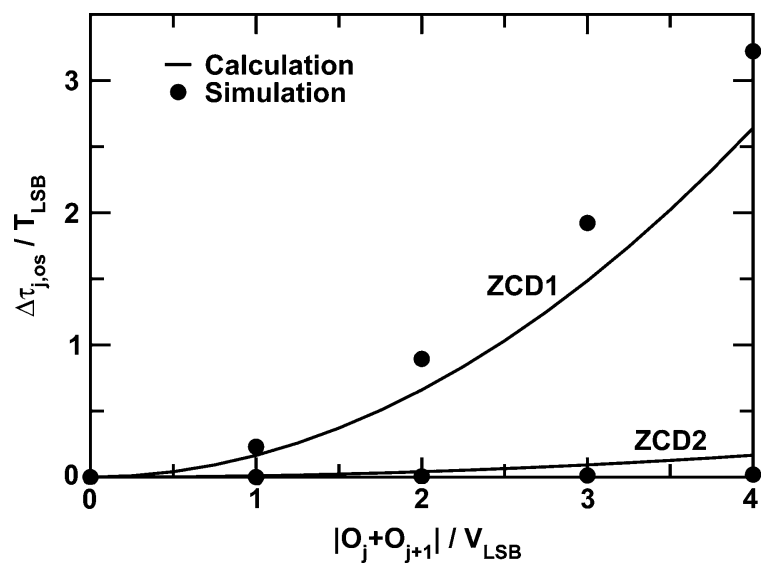

Fig. 8. Skew measurement error due to comparators' offsets. The input is a high-frequency sinewave with $f_{i} \approx 3.75 f_{c}$.

$\left|O_{j}-O_{j+1}\right|$ are normalized with $T_{\mathrm{LSB}}$ and $V_{\mathrm{LSB}}$ respectively, where $T_{\mathrm{LSB}}$ and $V_{\mathrm{LSB}}$ are defined in Section I. To achieve $\Delta \tau_{j, o s}<1 T_{\mathrm{LSB}}$, the offset requirement for the ZCD1 is $\left|O_{j}-O_{j+1}\right|<1.27 V_{\mathrm{LSB}}$. On the other hand, to illustrate a high $f_{i}$ case, let $f_{i}=\left(983057 / 2^{18}\right) f_{c} \approx 3.75 f_{c}$, then we have $Z_{R}=2 f_{i} \approx 7.5 f_{c}, \rho_{j, j+1}=-0.98$, and $\sigma_{j}^{2}=1 / 2$. The resulting $\Delta \tau_{j, o s}$ versus $\left|O_{j}+O_{j+1}\right|$ is shown in Fig. 8. To achieve $\Delta \tau_{j, o s}<1 T_{\mathrm{LSB}}$, the offset requirement for the ZCD1 is $\left|O_{j}+O_{j+1}\right|<2.4 V_{\mathrm{LSB}}$. Although its offset requirement is more stringent than that of a high $f_{i}$ case, a low $f_{i}$ case is usually preferred. A lower $f_{i}$ frequency implies a slower (and hence lower power) circuit for realizing the $x(t)$ signal generator. In addition, the $x(t)$ signal leakage due to capacitor coupling is less severe if a lower $f_{i}$ is chosen.

The preceding analyses assume that the mean value of the $x(t)$ signal is zero. The effect of $x(t)$ 's dc offset is also analyzed in Appendix A. As described in (26) and (27), both $P_{j, j+1}^{z}$ and $Z_{R}$ are reduced by the dc offset, $O_{x}$. Since its effect is identical to all ZC detectors in the proposed timing-skew detection scheme, the $O_{x}$ does not affect its accuracy. As described in the next section, timing skew is detected by measuring the $P_{j, j+1}^{z}$ difference between the $\mathrm{ZC}$ detectors. The absolute value of $P_{j, j+1}^{z}$ is irrelevant. 


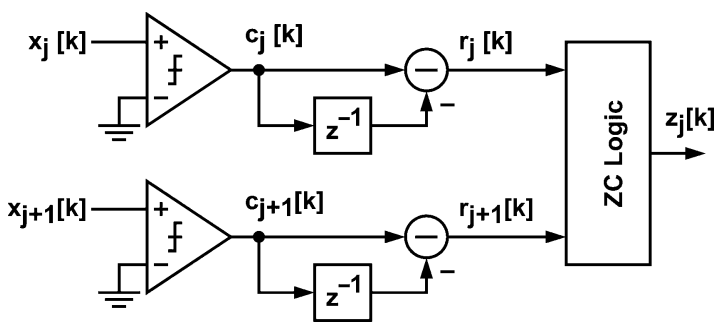

Fig. 9. Pseudo ZC detector (ZCD2).

\section{B. Pseudo ZC Detector (ZCD2)}

Fig. 9 shows the proposed pseudo ZC detector (ZCD2). Two $1-z^{-1}$ high-pass filters are added after the comparators to reduce the detection sensitivity to comparators' offsets. Outputs from the comparators are $c_{j}[k]$ and $c_{j+1}[k]$. Their binary values are $\{0,1\}$. Thus, the outputs of the filters $r_{j}[k]$ and $r_{j+1}[k]$ have triple values $\{-1,0,+1\}$. The $\mathrm{ZC}$ logic determines the $z_{j}[k]$ output as follows. The output $z_{j}[k]=1$ if $r_{j}[k] \times r_{j+1}[k] \leq 0$, otherwise $z_{j}[k]=0$. In other words, $z_{j}[k]=0$ if both $r_{j}[k]$ and $r_{j+1}[k]$ are +1 or both are -1 .

To gain an insight of the ZCD2 operation, one can approximate the comparators as linear amplifiers with random quantization noises. Thus, the ZCD2 becomes a ZC detector that detects the ZC in the $x(t)-x\left(t-T_{c}\right)$ signal. The comparators' offsets are removed by the $1-z^{-1}$ operation, resulting in a reduced $\mathrm{ZC}$ detection sensitivity to offsets.

Unlike the ZCD1, whose output follows the ZC probability $P_{j, j+1}^{z}$ of (2) and the $\mathrm{ZC}$ density $Z_{R}\left(t_{0}, T_{c}\right)$ of (5), it is difficult to derive the explicit $P_{j, j+1}^{z}$ and $Z_{R}\left(t_{0}, T_{c}\right)$ expressions for the $\mathrm{ZCD} 2$. The ZCD2 detection is no longer a simple detection of $\mathrm{ZC}$ in $x(t)$. It detects certain events that cause $z_{j}[k]=1$. A simplified analysis for the cases with low $f_{i}$ frequency is provided in Appendix B. In such cases, $\rho_{j, j+1} \approx 1$, and the probability for $z_{j}[k]=1$ can be approximated by (36). As explained in Appendix B, the $Z_{R}\left(t_{0}, T_{c}\right)$ of (5) can be used to analyze the behaviors of both ZCD1 and ZCD2 in the low- $f_{i}$ scenario of the proposed timing-skew detection scheme. The effect of comparators' offsets on the ZCD2 is also analyzed in Appendix B. When offsets appear, the ZC probability $P_{j, j+1}^{z}$ is deviated from (36) by an amount of $\Delta P_{j, j+1}^{z}$. Its upper bound can be expressed as

$$
\Delta P_{j, j+1}^{z} \leq \frac{1}{4 \pi}\left(\frac{O_{j}}{\sigma_{j}}+\frac{O_{j+1}}{\sigma_{j+1}}\right)^{2} .
$$

Figs. 7 and 8 also show the ZCD2 timing skew measurement error due to the $O_{j}$ and $O_{j+1}$ offsets. Equation (10) is used for calculation data. It is clear from both figures that the ZCD2 is less sensitive to offsets. For the low- $f_{i}$ scenario with $f_{i} \approx$ $0.25 f_{c}$, the ZCD2 requires $\left|O_{j}+O_{j+1}\right|<3.1 V_{\mathrm{LSB}}$ to achieve a skew measurement error less than $1 T_{\mathrm{LSB}}$.

\section{Multiphase Timing-SKew CALibRation}

Consider the $M$-phase sampling system of Fig. 1. It samples the $x(t)$ reference and generates the $x_{j}[k]$ of $(1)$, where $1 \leq$ $j \leq M$. The sampling interval between $x_{j}[k]$ and $x_{j+1}[k]$ is $T_{s}-\tau_{j}+\tau_{j+1}$. A timing skew occurs if $\tau_{j} \neq \tau_{j+1}$. Fig. 2 shows the proposed multiphase timing-skew calibration architecture.

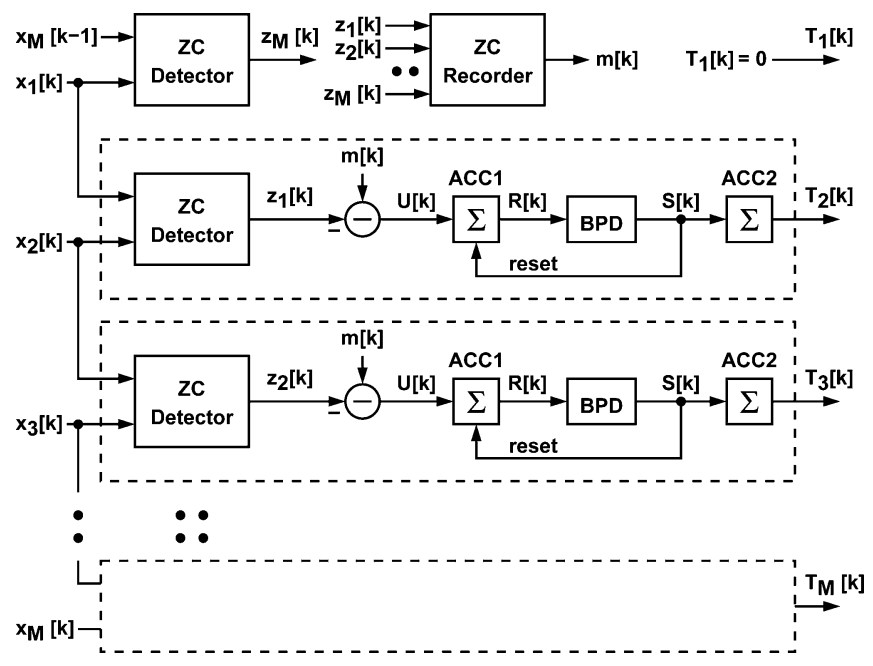

Fig. 10. Multiphase timing-skew calibration processor.

A multiphase timing-skew calibration processor (CP) is used to detect the timing skew between every adjacent clock pair. For each $j$, the delay of the $B_{j}$ clock buffer is controlled by the $T_{j}[k]$ output from the $\mathrm{CP}$, such that

$$
\tau_{j}[k]=\tau_{j, 0}+\mu_{t} \times T_{j}[k]
$$

where $\mu_{t}$ is the step size for the timing control and $\tau_{j, 0}$ is the timing offset of the $\phi_{j}$ clock when $T_{j}[k]=0$. The CP measures the timing skew between $\phi_{j}$ and $\phi_{j+1}$ and then adjusts $T_{j+1}[k]$ to minimize the skew.

Fig. 10 shows the CP's block diagram. It includes $M$ ZC detectors, which are deployed to measure the sampling intervals. Either the ZCD1 of Fig. 6 or the ZCD2 of Fig. 9 can be used as the $\mathrm{ZC}$ detectors. The $x(t)$ reference is assumed to be a narrowband signal. Its center frequency $f_{i}$ is near $0.25 f_{c}$ in order to establish a low- $f_{i}$ scenario. For the $j$ th calibration channel, its ZC detector senses any ZC between $x_{j}[k]$ and $x_{j+1}[k]$, and generates a binary output, $z_{j}[k] \in\{0,1\}$. The probability of $z_{j}[k]=1$ is $P_{j, j+1}^{z}$, which can be calculated from the ZC density $Z_{R}\left(t_{0}, T_{c}\right)$ using (6). From (7), a narrowband asynchronous $x(t)$ reference has a uniform $Z_{R}\left(t_{0}, T_{c}\right)$ close to $2 f_{i}$. Thus, the $P_{j, j+1}^{z}$ probability is proportional to the sampling interval between $x_{j}[k]$ and $x_{j+1}[k]$, which is denoted the $j$-interval. In Fig. 10, the $z_{j}[k]$ sequence is integrated onto an ACC 1 accumulator. The accumulator's output represents the average of $z_{j}[k]$, which is also proportional to the $j$-interval.

For each $j$, the CP compares the $j$-interval with the nominal sampling interval. The difference between the two intervals is the timing skew. The $\mathrm{CP}$ then adjusts $T_{j+1}[k]$ to minimize the skew. The nominal sampling interval is defined as the average of all $j$-intervals where $1 \leq j \leq M$. In Fig. 10, the timing skew is calculated as the difference between the accumulation of $z_{j}[k]$ and the accumulation of $m[k]$. The $m[k]$ sequence represents the average of the $\mathrm{ZC}$ occurrences among all sampling intervals. The $m[k]$ is generated from the ZC recorder shown in Fig. 11. The recorder accumulates every ZC from all ZC detectors. A comparator compares the accumulation result $a[k]$ with $M$, and generates a binary $m[k] \in\{0,1\}$ for every clock cycle. Whenever $a[k] \geq M$, the comparator issues $m[k]=1$, and an amount 


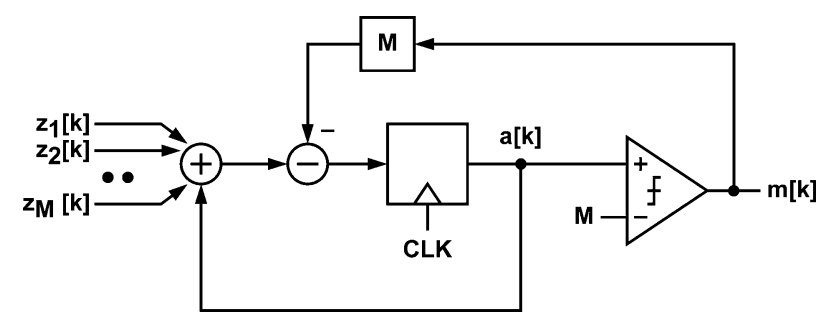

Fig. 11. ZC recorder.

of $M$ is subtracted from the accumulation result during the following clock cycle. Note that $m[k]$ is a sequence of 0 and 1 . Its mean value represents the nominal sampling interval. The operation of $m[k]$ averaging is provided by the ACC1 accumulator in each calibration channel. The proposed ZC recorder is simple and its hardware cost is low. Note that the extra ZC detector in the upper left corner of Fig. 10 detects the $\mathrm{ZC}$ occurrences in the sampling interval between $\phi_{M}$ and $\phi_{1}$. This sampling interval is not measured by the calibration channels that generate $T_{2}[k]$, $T_{3}[k], \ldots, T_{M}[k]$.

In the $j$ th calibration channel, the timing skew is calculated as $U[k]=m[k]-z_{j}[k]$. A simple calibration loop can be formed by applying $T_{j+1}[k+1]=T_{j+1}[k]+\mu \times U[k]$ with $\mu<1$. However, the resulting fluctuation in $T_{j+1}[k]$ is large unless a very small $\mu$ is chosen. In Fig. 10, an additional accumulator is added to reduce the $T_{j+1}[k]$ fluctuation. The ACC1 accumulator integrates the $U[k]$ sequence, and generates the $R[k]$ output. A bilateral peak detector, BPD, monitors the value of $R[k]$ and generates a corresponding triple-valued output $S[k] \in$ $\{+1,0,-1\}$. The BPD has two thresholds $+N_{C}$ and $-N_{C}$. Whenever $R[k] \geq+N_{C}, S[k]=+1$. Whenever $R[k] \leq-N_{C}$, $S[k]=-1$. Otherwise, $S[k]=0$. In addition, the ACC1 accumulator is reset to zero whenever $S[k]=+1$ or $S[k]=-1$. Thus, $-N_{C} \leq R[k] \leq+N_{C}$. Each time $S[k]$ is either +1 or -1 , it can retain such state for only one clock cycle. Finally, the ACC2 accumulator integrates the $S[k]$ sequence and generates the $T_{j+1}[k]$ output. By adding the integration-and-dump operation of ACC1 and BPD, the fluctuation in $T_{j+1}[k]$ is reduced.

In the proposed calibration scheme, the $\phi_{1}$ clock in Fig. 2 is a designated reference phase. There is no need to adjust the corresponding $T_{1}[k]$ control. Thus, $T_{1}[k]$ is preset to 0 in the $\mathrm{CP}$ of Fig. 10.

The proposed calibration scheme contains two system parameters, $\mu_{t}$ and $N_{C}$. To simplify analysis, we assume each calibration channel in the CP of Fig. 10 employs identical $\mu_{t}$ and $N_{C}$. Together with the $Z_{R}$ of $x(t)$, they affect calibration behaviors, such as calibration converging speed and timing fluctuation. In general, large $\mu_{t}$ and small $N_{C}$ result in fast converging speed but large timing fluctuation. On the other hand, small $\mu_{t}$ and large $N_{C}$ result in small timing fluctuation but also slow converging speed. The following two subsections give detailed analyses.

\section{A. Convergence Speed}

Consider the $j$ th calibration channel in Fig. 10. Its ZC detector measures the sampling interval between the $\phi_{j}$ and $\phi_{j+1}$ clocks. According to (1), the $\phi_{j}$ and $\phi_{j+1}$ clocks have the timing

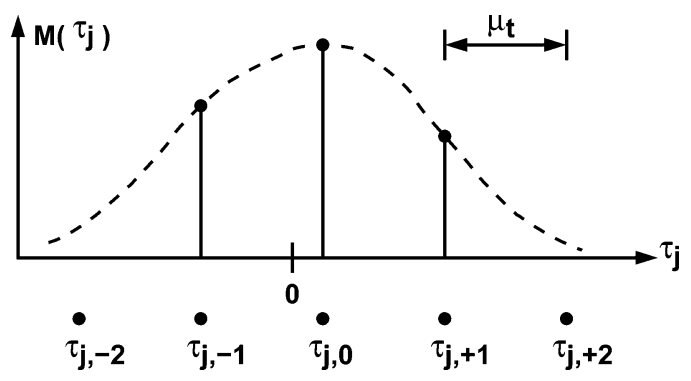

Fig. 12. Probability mass function of $\tau_{j}, M\left(\tau_{j}\right)$.

offsets $\tau_{j}$ and $\tau_{j+1}$, respectively. In Fig. 10, the $U[k]$ signal is the difference between $z_{j}[k]$ and $m[k]$, representing the timing skew $\tau_{j}-\tau_{j+1}$. The $U[k]$ is used to update the $T_{j+1}[k]$ signal, which controls the $\tau_{j+1}[k]$ timing offset. In most cases, this calibration loop can be modeled as a continuous-time single-pole feedback system like

$$
d \tau_{j+1}=\mu_{t} \times \frac{\left(\tau_{j}-\tau_{j+1}\right) \cdot Z_{R} \cdot d k}{N_{C}} .
$$

The above equation states that, to update $\tau_{j+1}$ by one $\mu_{t}$ step, it takes $d k$ sampling intervals during which the $\tau_{j}-\tau_{j+1}$ timing skew causes $N_{C}$ ZCs. Thus, we obtain the following differential equation for $\tau_{j+1}[k]$ :

$$
\frac{d \tau_{j+1}[k]}{d k}=-\frac{\tau_{j+1}[k]-\tau_{j}[k]}{\tau_{c}}
$$

where the system time constant $\tau_{c}$ is

$$
\tau_{c}=\frac{N_{C}}{\mu_{t}} \times \frac{1}{Z_{R}} .
$$

For a $M$-channel system, (12) with $1 \leq j \leq M-1$ can be expanded into $M-1$ coupled equations. In most practical cases, $\tau_{c}$ is much larger than 1 . Thus, by treating $\tau_{j}[k]$ as a constant, the transient behavior of $\tau_{j+1}[k]$ can be approximated by a simple exponential function with the $\tau_{c}$ time constant.

\section{B. Timing Fluctuation}

Consider the $\tau_{j}[k]$ of (11). The CP measures the sampling interval between the $\phi_{j-1}$ and $\phi_{j}$ clocks, and then adjusts $T_{j}[k]$. Assume $T_{j-1}[k]$ remains constant and $\phi_{j-1}$ is fixed. The CP adjusts only $T_{j}[k]$ to force $\tau_{j}[k]$ moving toward 0 . As the process converges, the behavior of $\tau_{j}[k]$ becomes a discrete random fluctuation around zero. Fig. 12 illustrates a probability mass function for $\tau_{j}, M\left(\tau_{j}\right)$. The discrete values for $\tau_{j}$ is $\tau_{j, 0}, \tau_{j, \pm 1}$, $\tau_{j, \pm 2}, \ldots$, with $\tau_{j, 0}$ being closest to zero. The distance between two adjacent discrete values is $\mu_{t}$. The value of $\tau_{j, 0}$ is between $-0.5 \mu_{t}$ and $+0.5 \mu_{t}$. The calibration loop forces the maximum value of $M\left(\tau_{j}\right)$ to occur at $\tau_{j, 0}$. A mathematical treatment of $M\left(\tau_{j}\right)$ is included in Appendix C. The resulting standard deviation of $\tau_{j}$, averaged over possible value of $\tau_{j, 0}$, can be found as

$$
\sigma^{2}(\tau)=\frac{1}{6} \mu_{t}^{2}+\frac{4 T_{s}}{3 N_{C}} \mu_{t} .
$$

Obviously, smaller $\mu_{t}$ and larger $N_{C}$ can reduce $\sigma^{2}(\tau)$.

For the multiphase calibration system shown in Figs. 2 and 10 , the $\phi_{1}$ clock with $T_{1}[k]=0$ is chosen as the designated reference phase. All other clocks are adjusted by the CP to achieve 


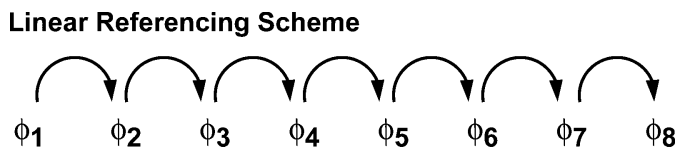

Circular Referencing Scheme

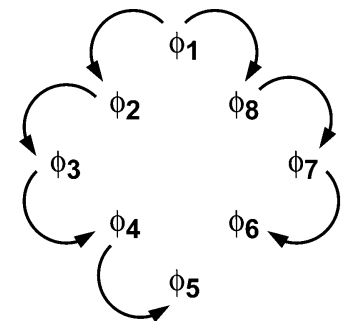

Fig. 13. Referencing schemes for multiphase clocks.

uniform phase spacing. The timing skew between $\phi_{1}$ and $\phi_{2}$ is minimized by adjusting the delay of the $\phi_{2}$ clock buffer through $T_{2}[k]$. The timing skew between $\phi_{2}$ and $\phi_{3}$ is minimized by adjusting the delay of the $\phi_{3}$ clock buffer through $T_{3}[k]$. This calibration arrangement repeats for $\phi_{4}, \phi_{5}$, and so on and is referred to as the linear referencing arrangement illustrated in Fig. 13. Note that $\phi_{1}$ does not fluctuate. The timing fluctuation of $\phi_{2}$ is summarized by (14). The timing fluctuation of $\phi_{3}$ is larger than (14), since it uses the fluctuating $\phi_{2}$ as its phase reference. In fact, the timing fluctuation is accumulated along the reference chain. The standard deviation of the $\phi_{j}$ 's timing fluctuation can be expressed as

$$
\sigma^{2}\left(\tau_{j}\right)=(j-1) \times \sigma^{2}(\tau) \quad j \geq 2 .
$$

To reduce the overall timing fluctuation, the circular referencing arrangement shown in Fig. 13 is suggested. In this scheme, both $\phi_{2}$ and $\phi_{8}$ use $\phi_{1}$ as the reference for timing-skew calibration. Then, $\phi_{3}$ and $\phi_{7}$ use $\phi_{2}$ and $\phi_{8}$ as the references, respectively. In this arrangement, the maximum $\sigma^{2}\left(\tau_{j}\right)$ is reduced by half. The overall averaged timing fluctuation is

$$
\sigma^{2}\left(\tau_{T}\right) \equiv \frac{1}{M} \sum_{j=1}^{M} \sigma^{2}\left(\tau_{j}\right)=\frac{M}{4} \sigma^{2}(\tau)=\frac{M}{24} \mu_{t}^{2}+\frac{M T_{s}}{3 N_{C}} \mu_{t}
$$

where $M$ is assumed to be an even number. For this multiphase calibration system, $\sigma^{2}\left(\tau_{T}\right)$ is proportional to the number of phases, $M$.

To realize the circular referencing arrangement shown in Fig. 13, the calibration processor of Fig. 10 is modified as follows. The calibration channel that generates the $T_{8}[k]$ output takes $x_{1}[k]$ and $x_{8}[k]$ as its inputs. The calibration channel that generates the $T_{7}[k]$ output takes $x_{8}[k]$ and $x_{7}[k]$ as its inputs. The calibration channel that generates the $T_{6}[k]$ output takes $x_{7}[k]$ and $x_{6}[k]$ as its inputs. The calibration channels for $T_{1}[k], \ldots, T_{5}[k]$ remain unchanged. The extra ZC detector at the upper left corner of Fig. 10 now receives $x_{5}[k]$ and $x_{6}[k]$ as its inputs and generates the $z_{5}[k]$ output.

\section{EIGHT-ChANNEL TI ADC EXAMPLE}

Fig. 14 shows an eight-channel TI ADC that employs the timing-skew calibration scheme described in Section IV. There are eight $\mathrm{A} / \mathrm{D}$ channels, i.e., $\mathrm{ADC}_{j}$, where $1 \leq j \leq 8$. Each

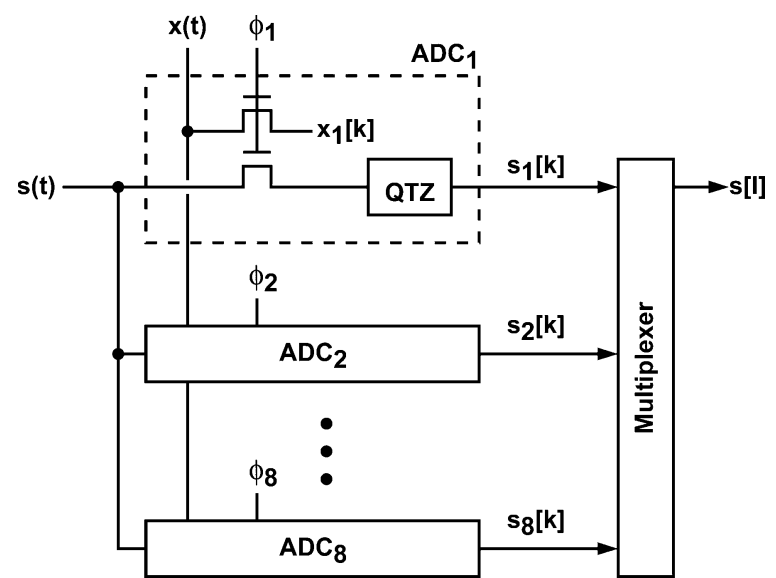

Fig. 14. Eight-channel TI ADC.

A/D channel samples and quantizes the $s(t)$ input and produces a corresponding $s_{j}[k]$ sequence. The $s_{j}[k]$ sequences from all channels are then multiplexed to generate the final $s[l]$ digital output. In the $j$ th channel, there are two samplers driven by the same $\phi_{j}$ clock. One sampler samples the $s(t)$ input to be quantized into $s_{j}[k]$. The succeeding quantizer (QTZ) has 6-b resolution and an input range between \pm 1 . The other sampler receives the $x(t)$ reference and generates the $x_{j}[k]$ sequence. The $x_{j}[k]$ sequence is sent to the timing-skew calibration processor shown in Fig. 10. Its $T_{j}[k]$ output controls the $B_{j}$ clock buffer shown in Fig. 2. For the eight-phase clock generator, the nominal timing interval between two adjacent phases is $T_{s}$. Each $\phi_{j}$ clock has a period of $T_{c}=8 T_{s}$ and a frequency of $f_{c}=1 / T_{c}$. The entire system is equivalent to a 6-b ADC with a sampling rate of $f_{s}=1 / T_{s}=8 f_{c}$. As in Section I, we define the magnitude resolution as $V_{\mathrm{LSB}}=2 / 2^{6}=2^{-5}$ and the timing resolution as $T_{\mathrm{LSB}}=T_{s} / 2^{6}$.

In Fig. 14, each A/D channel includes a $s(t)$ sampler and a $x(t)$ sampler to enable the robust background timing-skew calibration. The two samplers driven by the same clock must have the same sampling instant. To minimize mismatch, the two samplers should be placed in close proximity in the chip layout. The signal routes of $s(t)$ and $x(t)$ may also have to be matched. Furthermore, the clock edges that define the sampling instants should be made as steep as necessary.

For timing-skew calibration, the narrowband $x(t)$ reference has a center frequency at $f_{i} \approx 0.25 f_{c}$, which meets the low- $f_{i}$ scenario described in Section III. From (7), the $x(t)$ reference has a ZC density of $Z_{R}=2 f_{i} \approx 0.5 f_{c}$. The resulting timing fluctuation variance averaged over the eight-phase clocks $\sigma^{2}\left(\tau_{T}\right)$ can be calculated using (16). Fig. 15 shows the relationship between $\sigma\left(\tau_{T}\right)$ and $N_{C}$ at various $\mu_{t}$ values. For this 6-b ADC example, $\mu_{t}=T_{s} / 2^{8}$ and $N_{C}=2^{10}$ are chosen to achieve $\sigma\left(\tau_{T}\right)=0.26 T_{\mathrm{LSB}}$.

The behavior of this TI ADC is simulated using a $\mathrm{C}$ program. To illustrate that the proposed calibration scheme does not require a well-defined sinewave, the $x(t)$ reference used in the simulation is a full-swing phase-modulated sinewave

$$
x(t)=\sin \left[2 \pi f_{i} t+0.1 \sin \left(2 \pi f_{m} t\right)\right]
$$




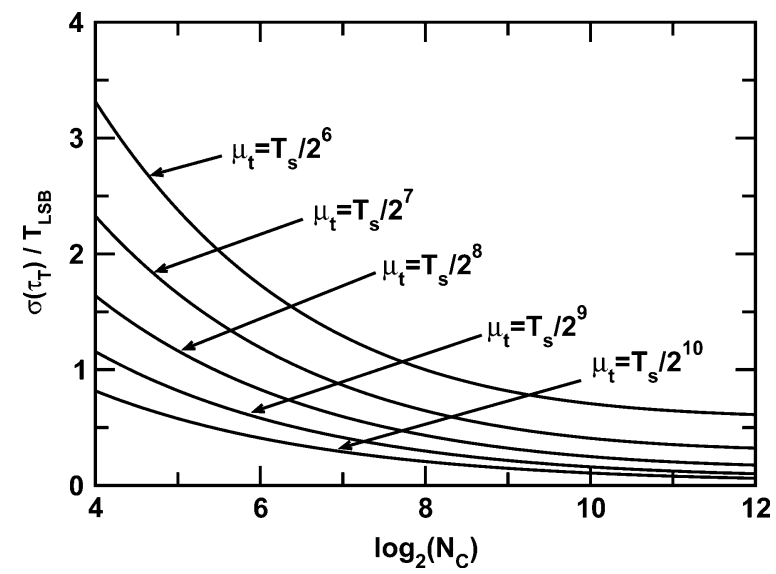

Fig. 15. Timing fluctuation $\sigma(\tau)$ versus $N_{C}$ and $\mu_{t}$ for the TI ADC example.

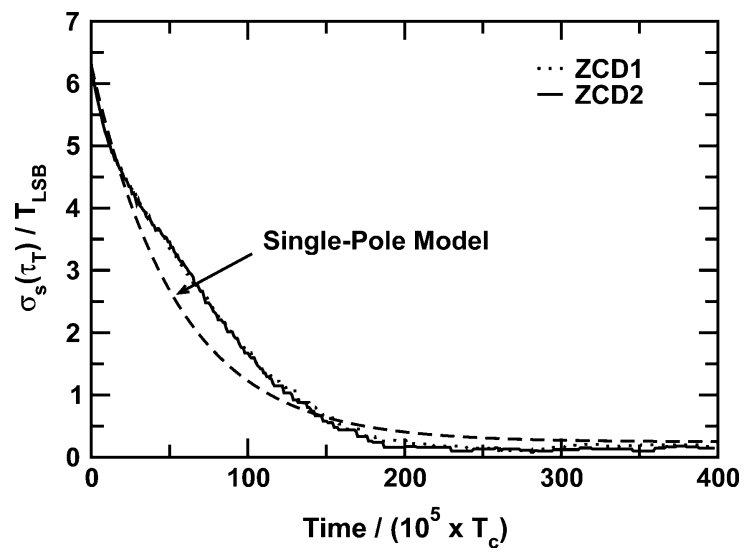

Fig. 16. Settling behavior of $\sigma_{s}\left(\tau_{T}\right)$. The comparators in ZCD1 and ZCD2 are ideal.

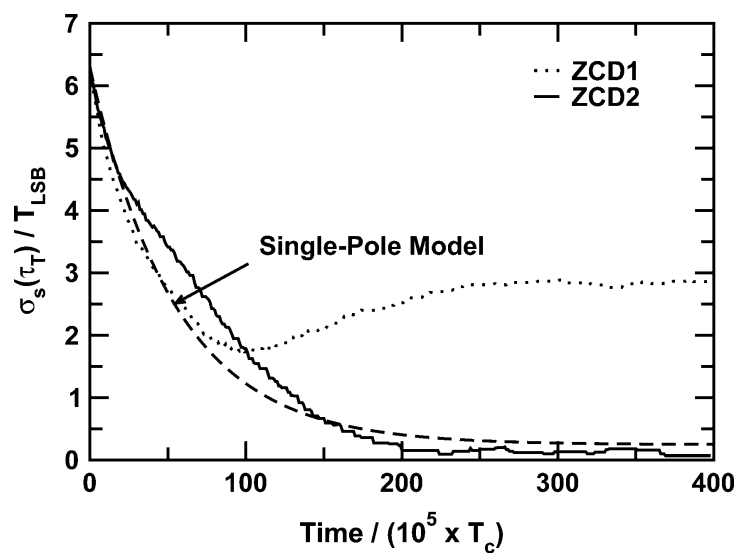

Fig. 17. Settling behavior of $\sigma_{s}\left(\tau_{T}\right)$. The comparators in ZCD1 and ZCD2 have random offsets. The standard deviation of the offsets is $\sigma\left(V_{O S}\right)=3 V_{\mathrm{LSB}}$.

where $f_{i}=\left(65519 / 2^{18}\right) f_{c} \approx 0.25 f_{c}$ and $f_{m}=$ $\left(36733 / 2^{18}\right) f_{c} \approx 0.14 f_{c}$. Both the ZCD1 and the ZCD2 described in Section III are tested. Fig. 16 shows the settling behavior of the ADC's timing-skew spatial standard deviation, $\sigma_{s}\left(\tau_{T}\right)$. For this figure, ideal comparators without offsets are deployed in the ZC detectors. Note that $\sigma_{s}\left(\tau_{T}\right)$ is the standard deviation of the $\left(\tau_{1}, \tau_{2}, \ldots, \tau_{8}\right)$ data set at a given time. For $1 \leq j \leq 8, \tau_{j}$ is the timing offset of the $\phi_{j}$ clock. A random

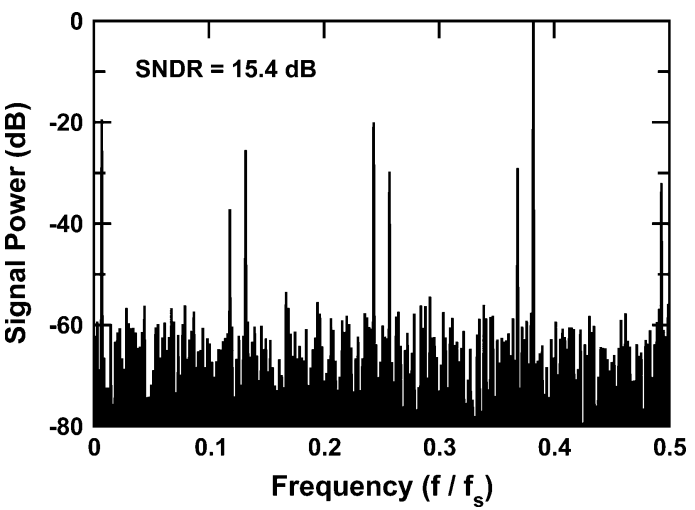

Fig. 18. Spectrum of $s[l]$ before timing-skew calibration.

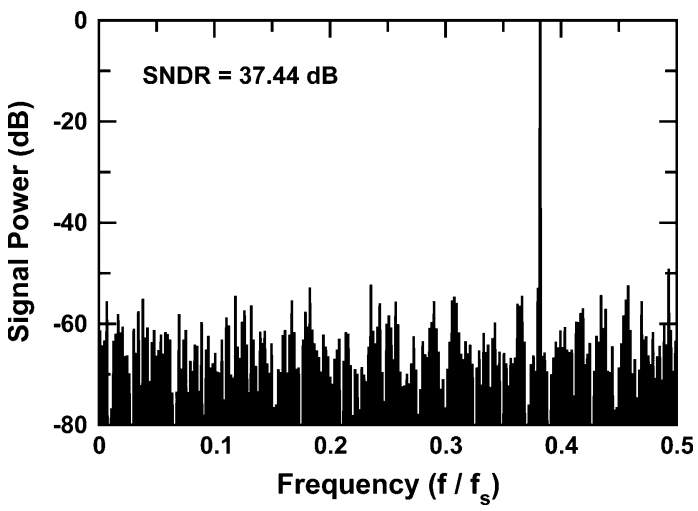

Fig. 19. Spectrum of $s[l]$ after timing-skew calibration.

number generator selects the initial value of $\tau_{j}$. The $\sigma_{s}\left(\tau_{T}\right)$ is $6.3 T_{\mathrm{LSB}}$ before calibration and settles toward $0.22 T_{\mathrm{LSB}}$ as calibration progresses. The $0.22 T_{\mathrm{LSB}}$ value is obtained by averaging $\sigma_{s}\left(\tau_{T}\right)$ over a period from $250 \times 10^{5} \times T_{c}$ to $400 \times 10^{5} \times T_{c}$. Without comparator offsets, ZCD1 and $\mathrm{ZCD} 2$ exhibit similar behavior. Also shown in the figure is the transient response of a single-pole model with a time constant $\tau_{c}=41.9 \times 10^{5} T_{c}$, which is calculated from (13). Fig. 17 shows the effect of comparators' offsets. Random offsets generated from a random number generator are added to the comparators in ZCD1 and ZCD2. The standard deviation of the offsets is $\sigma\left(V_{O S}\right)=3 V_{\mathrm{LSB}}$. For the calibration using ZCD1, the $\sigma_{s}\left(\tau_{T}\right)$ can only settle to $2.86 T_{\mathrm{LSB}}$. On the other hand, the $\sigma_{s}\left(\tau_{T}\right)$ can settle to $0.21 T_{\mathrm{LSB}}$ by using ZCD2. The comparators' offsets have little effect on ZCD2. Note that the $\sigma_{s}\left(\tau_{T}\right)$ performance from simulations is better than the prediction of (16). The timing fluctuation theory described in Appendix $\mathrm{C}$ is derived from random process, which assumes a scenario more random than those used in the simulations.

Figs. 18 and 19 show the spectra of the ADC's output, $s[l]$, before and after timing-skew calibration. The calibration processor uses the ZCD2 detectors for timing-skew detection. All parameters, including comparators' offsets and initial timing skews are identical to those used in Fig. 17. The $s(t)$ input is a sinewave with a frequency $(391 / 1024) f_{s} \approx 0.38 f_{s}$. The improvement by the calibration is obvious. The SNDR is improved from 15.4 to $37.44 \mathrm{~dB}$. 


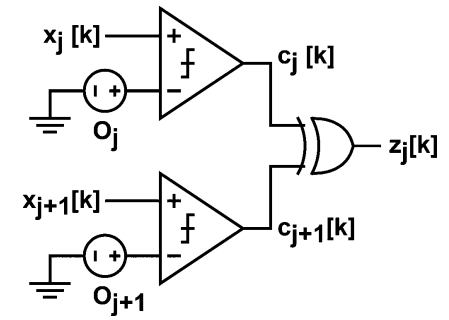

Fig. 20. ZCD1 with comparators' offsets.

\section{CONCLUSION}

The multiphase timing-skew calibration technique described in this paper is based on ZC detection. Although the ZC detection is a nonlinear operation, the $\mathrm{ZC}$ probability is related to the cross correlation of the calibration reference. We have made theoretical study on the $\mathrm{ZC}$ detection principle and proved its robustness. The ZCD2 with digital high-pass filters provides a detection technique that is less sensitive to comparators' offsets. With proper reference input, the ZC detectors can be used to measure the phase spacing among multiphase clocks. The $\mathrm{ZC}$ probability is proportional to the phase spacing. Based on this fact, we propose a digital calibration processor. It receives outputs from the $\mathrm{ZC}$ detectors and adjusts the delays of clock buffers to equalize the phase spacings between adjacent clock pairs. We have also analyzed the calibration processor's behaviors, including converging speed and timing fluctuation.

An eight-channel 6-b TI ADC example is used to demonstrate an application of the proposed calibration technique. Simulations of the ADC show that the results from the theoretical studies can correctly predict the calibration behaviors. Although the proposed calibration scheme needs an extra reference signal, the requirement for this reference is loose. The reference does not need to have an accurate frequency or an exact waveform shape. It can be easily generated on a chip using simple circuitry. Most of the calibration procedures are performed in the digital domain, and require only adders and registers.

\section{APPENDIX A \\ MATHEMATICAL ANALYSIS OF ZCD1}

Fig. 20 shows a ZCD1 whose two comparators exhibit $O_{j}$ and $O_{j+1}$ offsets, respectively. The $x_{j}[k]$ and $x_{j+1}[k]$ are two $x(t)$ sampling sequences corresponding to the $\phi_{j}$ and $\phi_{j+1}$ sampling clocks, respectively. Let $x(t)$ be a stationary Gaussian process with zero mean. Then, $x_{j}[k]$ and $x_{j+1}[k]$ form a bivariate normal distribution. Its probability density function is [10], [11]

$$
p_{b}=\frac{\exp \left[\frac{-\gamma}{\left[2\left(1-\rho_{j, j+1}^{2}\right)\right]}\right]}{2 \pi \sqrt{1-\rho_{j, j+1}^{2}} \times \sigma_{j} \sigma_{j+1}}
$$

with

$$
\gamma=\frac{x_{j}^{2}}{\sigma_{j}^{2}}+\frac{x_{j+1}^{2}}{\sigma_{j+1}^{2}}-\frac{2 \rho_{j, j+1} \cdot x_{j} x_{j+1}}{\sigma_{j} \sigma_{j+1}}
$$

where $\sigma_{j}$ and $\sigma_{j+1}$ are standard deviations as defined in (4) and $\rho_{j, j+1}$ the cross correlation between $x_{j}[k]$ and $x_{j+1}[k]$ as defined in (3).

In Fig. 20, the comparators' outputs, $c_{j}[k]$ and $c_{j+1}[k]$, are determined by the $x_{j}[k]-O_{j}$ polarity and $x_{j+1}[k]-O_{j+1}$ polarity, respectively. The $z_{j}[k]$ output is 1 only if $(1) x_{j}[k]>O_{j}$ and $x_{j+1}[k]<O_{j+1}$; or $(2) x_{j}[k]<O_{j}$ and $x_{j+1}[k]>O_{j+1}$. The probability of the first condition is

$$
\begin{aligned}
P_{1}= & \int_{-\infty}^{O_{j}} \int_{O_{j+1}}^{\infty} p_{b} d x_{j+1} d x_{j} \\
= & \int_{-\infty}^{0} \int_{0}^{\infty} p_{b} d x_{j+1} d x_{j} \\
& +\left(\int_{0}^{O_{j}} \int_{0}^{\infty}-\int_{-\infty}^{0} \int_{0}^{O_{j+1}}-\int_{0}^{O_{j}} \int_{0}^{O_{j+1}}\right) p_{b} d x_{j+1} d x_{j}
\end{aligned}
$$

In the last equation, the first term on the right-hand side is the probability with $O_{j}=O_{j+1}=0$, and the second term is the effect of offsets. Similarly, the probability of the second condition is

$$
\begin{aligned}
P_{2}= & \int_{O_{j}}^{\infty} \int_{-\infty}^{O_{j+1}} p_{b} d x_{j+1} d x_{j} \\
= & \int_{0}^{\infty} \int_{-\infty}^{0} p_{b} d x_{j+1} d x_{j} \\
& +\left(-\int_{0}^{O_{j}} \int_{-\infty}^{0}+\int_{0}^{\infty} \int_{0}^{O_{j+1}}-\int_{0}^{O_{j}} \int_{0}^{O_{j+1}}\right) \\
& \times p_{b} d x_{j+1} d x_{j} .
\end{aligned}
$$

Thus, the total probability of $z_{j}[k]=1$ is

$$
P_{j, j+1}^{z}\left(O_{j}, O_{j+1}\right)=P_{1}+P_{2}=P_{j, j+1}^{z}+\Delta P_{j, j+1}^{z},
$$

where $P_{j, j+1}^{z}$ as defined in (2) is the ideal ZC probability, and

$$
\begin{aligned}
\Delta P_{j, j+1}^{z} \approx & \frac{\rho_{j, j+1}}{2 \pi \sqrt{1-\rho_{j, j+1}^{2}}} \\
& \quad \times\left(\frac{O_{j}^{2}}{\sigma_{j}^{2}}+\frac{O_{j+1}^{2}}{\sigma_{j+1}^{2}}-\frac{2}{\rho_{j, j+1}} \frac{O_{j}}{\sigma_{j}} \frac{O_{j+1}}{\sigma_{j+1}}\right)
\end{aligned}
$$

Equation (25), as repeated in (8), is the effect of offsets.

Consider a $x(t)$ signal having a non-zero dc component. It is equivalent to the case that both comparators in ZCD1 exhibit identical offsets, i.e., $O_{j}=O_{j+1}=O_{x}$. From (24) and (25), the corresponding $\mathrm{ZC}$ probability becomes

$$
P_{j, j+1}^{z} \approx \frac{1}{2}-\frac{1}{\pi} \sin ^{-1} \rho_{j, j+1}-\frac{1-\rho_{j, j+1}}{\pi \sqrt{1-\rho_{j, j+1}^{2}}} \frac{O_{x}^{2}}{\sigma_{j}^{2}} .
$$

And the zero crossing rate becomes

$$
Z_{R}\left(t_{0}, T_{c}\right)=\left.\frac{1}{\pi}\left(1-\frac{O_{x}^{2}}{2 \sigma_{j}^{2}}\right) \sqrt{\frac{-\partial^{2} \rho_{a, b}\left(t_{0}, T_{c}, t_{s}\right)}{\partial t_{s}^{2}}}\right|_{t_{s}=0} .
$$

Both $P_{j, j+1}^{z}$ and $Z_{R}$ are reduced by $O_{x}^{2}$. 


\section{APPENDIX B}

MATHEMATICAL ANALYSIS OF ZCD2

Consider the ZCD2 shown in Fig. 9. To simplify denotation, define $x_{j}[k]=x_{1}, x_{j}[k-1]=x_{2}, x_{j+1}[k]=x_{3}$, and $x_{j+1}[k-$ 1] $=x_{4}$.

First, consider the case of three random variables $x_{1}, x_{2}$, and $x_{3}$. Assume they form the trivariate normal distribution with given cross correlation $\rho_{i, j}$ as defined (3), where $\{i, j\} \in 1,2,3$. Define $P_{a}, P_{b}, P_{c}$, and $P_{d}$ probabilities as

$$
\begin{aligned}
& P_{a} \equiv P\left(x_{1}<0, x_{2}<0, x_{3}<0\right)=P\left(x_{1}>0, x_{2}>0, x_{3}>0\right) \\
& P_{b} \equiv P\left(x_{1}<0, x_{2}>0, x_{3}<0\right)=P\left(x_{1}>0, x_{2}<0, x_{3}>0\right) \\
& P_{c} \equiv P\left(x_{1}>0, x_{2}<0, x_{3}<0\right)=P\left(x_{1}<0, x_{2}>0, x_{3}>0\right) \\
& P_{d} \equiv P\left(x_{1}>0, x_{2}>0, x_{3}<0\right)=P\left(x_{1}<0, x_{2}<0, x_{3}>0\right) .
\end{aligned}
$$

Obviously, $P_{a}+P_{b}+P_{c}+P_{d}=1 / 2$, and

$$
\begin{aligned}
& P_{a}+P_{b}=P\left(x_{1}<0, x_{3}<0\right)=\frac{1}{4}+\frac{\sin ^{-1} \rho_{1,3}}{2 \pi} \\
& P_{a}+P_{c}=P\left(x_{2}<0, x_{3}<0\right)=\frac{1}{4}+\frac{\sin ^{-1} \rho_{2,3}}{2 \pi} \\
& P_{a}+P_{d}=P\left(x_{1}<0, x_{2}<0\right)=\frac{1}{4}+\frac{\sin ^{-1} \rho_{1,2}}{2 \pi}
\end{aligned}
$$

where $\rho_{1,3}, \rho_{2,3}$, and $\rho_{1,2}$ are correlations between $x_{1}$ and $x_{3}$, $x_{2}$ and $x_{3}$, and $x_{1}$ and $x_{2}$, respectively. The above equations can be solved to give

$$
\begin{aligned}
& P_{a}=\frac{1}{8}+\frac{1}{4 \pi}\left(\sin ^{-1} \rho_{1,2}+\sin ^{-1} \rho_{1,3}+\sin ^{-1} \rho_{2,3}\right) \\
& P_{b}=\frac{1}{8}+\frac{1}{4 \pi}\left(-\sin ^{-1} \rho_{1,2}+\sin ^{-1} \rho_{1,3}-\sin ^{-1} \rho_{2,3}\right) \\
& P_{c}=\frac{1}{8}+\frac{1}{4 \pi}\left(-\sin ^{-1} \rho_{1,2}-\sin ^{-1} \rho_{1,3}+\sin ^{-1} \rho_{2,3}\right) \\
& P_{d}=\frac{1}{8}+\frac{1}{4 \pi}\left(\sin ^{-1} \rho_{1,2}-\sin ^{-1} \rho_{1,3}-\sin ^{-1} \rho_{2,3}\right) .
\end{aligned}
$$

Now consider the case of four random variables $x_{1}, x_{2}, x_{3}$, and $x_{4}$. It is difficult to solve the probabilities in the form of (30). Simplified solution is provided for the low-frequency case as follows. Define $P_{A}, P_{B}$, and $P_{c}$ as

$$
\begin{aligned}
& P_{A} \equiv P\left(x_{1}<0, x_{2}>0, x_{3}<0, x_{4}>0\right) \\
& P_{B} \equiv P\left(x_{1}<0, x_{2}>0, x_{3}>0, x_{4}<0\right) \\
& P_{C} \equiv P\left(x_{1}<0, x_{2}>0, x_{3}>0, x_{4}>0\right) .
\end{aligned}
$$

From (30), we have

$$
\begin{aligned}
P_{A}+P_{C}= & P\left(x_{1}<0, x_{2}>0, x_{4}>0\right) \\
= & \frac{1}{8}+\frac{1}{4 \pi}\left(-\sin ^{-1} \rho_{1,2}\right. \\
& \left.\quad-\sin ^{-1} \rho_{1,4}+\sin ^{-1} \rho_{2,4}\right) \\
P_{B}+P_{C}= & P\left(x_{1}<0, x_{2}>0, x_{3}>0\right) \\
= & \frac{1}{8}+\frac{1}{4 \pi}\left(-\sin ^{-1} \rho_{1,2}\right. \\
& \left.\quad-\sin ^{-1} \rho_{1,3}+\sin ^{-1} \rho_{2,3}\right) .
\end{aligned}
$$

For the low-frequency case, $\rho_{1,3}=\rho_{2,4} \approx 1$ and $P_{B} \approx 0$. As a result, we can solve the above equations and obtain

$P_{A}=-\frac{1}{4 \pi}\left(\sin ^{-1} \rho_{1,4}+\sin ^{-1} \rho_{2,3}-\sin ^{-1} \rho_{1,3}-\sin ^{-1} \rho_{2,4}\right)$.

Then, the probability of $z_{j}[k]=1$ is

$$
\begin{aligned}
P_{j, j+1}^{z}= & 1-2 P_{A} \\
= & 1+\frac{1}{2 \pi}\left(\sin ^{-1} \rho_{1,4}+\sin ^{-1} \rho_{2,3}\right. \\
& \left.-\sin ^{-1} \rho_{1,3}-\sin ^{-1} \rho_{2,4}\right) .
\end{aligned}
$$

The above equation can be rewritten as

$$
\begin{aligned}
P_{j, j+1}^{z}=\left.\frac{1}{\pi} \sin ^{-1} \rho_{a, b}\left(t_{0}, T_{c}, t_{s}\right)\right|_{t_{s}=T_{c}} \\
+\int_{t_{j}}^{t_{j}+T_{s}} Z_{R}\left(t_{0}, T_{c}\right) d t_{0}
\end{aligned}
$$

where $Z_{R}\left(t_{0}, T_{c}\right)$ is defined in (5). The $Z_{R}\left(t_{0}, T_{c}\right)$ is identical to the $Z_{R}\left(t_{0}, T_{c}\right)$ for ZCD1. Note that the first term on the righthand side of (36) is independent of $T_{s}$. Only the second term containing $Z_{R}\left(t_{0}, T_{c}\right)$ is relevant in the proposed timing-skew detection scheme. Thus, we state that both ZCD1 and ZCD2 show identical $Z_{R}\left(t_{0}, T_{c}\right)$ behavior in the proposed timing-skew detection scheme under the low- $f_{i}$ scenario.

Now consider a ZCD2 with internal comparators exhibiting offsets. The following assumption is made in order to simplify analysis. There are two different cases regarding the relationship between $x_{j}[k]$ and $x_{j}[k+1]$ : weak correlation and strong correlation. For the weak-correlation case, we can assume that the probability of $x_{1}=x_{j}[k]<0$ and $x_{3}=x_{j+1}[k]<0$ is independent of the probability of $x_{2}=x_{j}[k-1]>0$ and $x_{4}=x_{j+1}[k-1]>0$. Thus

$$
\begin{aligned}
P_{A} & \equiv P\left(x_{1}<0, x_{2}>0, x_{3}<0, x_{4}>0\right) \\
& \approx P\left(x_{1}<0, x_{3}<0\right) \times P\left(x_{2}>0, x_{4}>0\right) .
\end{aligned}
$$

Define probabilities $P_{1,3} \equiv P\left(x_{1}<0, x_{3}<0\right)$ and $P_{2,4} \equiv$ $P\left(x_{2}>0, x_{4}>0\right)$. When the comparators exhibit offsets, the corresponding probabilities becomes $P_{1,3}^{O}=P_{1,3}+\Delta P_{1,3}$ and $P_{2,4}^{O}=P_{2,4}+\Delta P_{2,4}$, where

$$
\begin{aligned}
\Delta P_{1,3}= & P\left(x_{1}<O_{j}, x_{3}<O_{j+1}\right)-P\left(x_{1}<0, x_{3}<0\right) \\
= & \int_{-\infty}^{O_{j}} \int_{-\infty}^{O_{j+1}} p_{b} d x_{3} d x_{1}-\int_{-\infty}^{0} \int_{-\infty}^{0} p_{b} d x_{3} d x_{1} \\
= & \left(\int_{0}^{O_{j}} \int_{-\infty}^{0}+\int_{-\infty}^{0} \int_{0}^{O_{j+1}}+\int_{0}^{O_{j}} \int_{0}^{O_{j+1}}\right) \\
& \times p_{b} d x_{3} d x_{1} \\
\approx & \frac{1}{2 \sqrt{2 \pi}}\left(\frac{O_{j}}{\sigma_{j}}+\frac{O_{j+1}}{\sigma_{j+1}}\right) \\
\Delta P_{2,4}= & P\left(x_{2}>O_{j}, x_{4}>O_{j+1}\right)-P\left(x_{2}>0, x_{4}>0\right) \\
\approx & -\frac{1}{2 \sqrt{2 \pi}}\left(\frac{O_{j}}{\sigma_{j}}+\frac{O_{j+1}}{\sigma_{j+1}}\right) \\
= & -\Delta P_{1,3} .
\end{aligned}
$$


Note that $P_{1,3}=P_{2,4}$. From (37) and using $P_{j, j+1}^{z}=1-2 P_{A}$, the $P_{j, j+1}^{z}$ probability for ZCD2 is deviated from (36) by

$$
\Delta P_{j, j+1}^{z}=2\left(\Delta P_{1,3}\right)^{2}=\frac{1}{4 \pi}\left(\frac{O_{j}}{\sigma_{j}}+\frac{O_{j+1}}{\sigma_{j+1}}\right)^{2} .
$$

The above equation is repeated in (10).

For the strong-correlation case, we assume that, under the $z_{j}[k]=1$ condition, the relationship between $x_{1}$ and $x_{2}$ is similar to the relationship between $x_{3}$ and $x_{4}$. Furthermore, $x_{1} \approx x_{3}$ or $x_{1} \approx-x_{3}$, and $x_{2} \approx x_{4}$ or $x_{2} \approx-x_{4}$. Thus,

$$
\begin{aligned}
P_{A} & \equiv P\left(x_{1}<0, x_{2}>0, x_{3}<0, x_{4}>0\right) \\
& \approx P\left(x_{1}<0, x_{2}>0\right) .
\end{aligned}
$$

The probability variation due to $O_{j}$ is

$$
\begin{aligned}
\Delta P_{A, O_{j}} & \equiv P\left(x_{1}<O_{j}, x_{2}>O_{j}\right)-P\left(x_{1}<0, x_{2}>0\right) \\
\text { or } & \equiv P\left(x_{1}>O_{j}, x_{2}<O_{j}\right)-P\left(x_{1}>0, x_{2}<0\right) \\
& =\frac{1}{\pi} \sqrt{\frac{1-\rho_{1,2}}{1+\rho_{1,2}}} \times \frac{O_{j}^{2}}{\sigma_{j}^{2}} .
\end{aligned}
$$

The probability variation due to $O_{j+1}$ is

$$
\begin{aligned}
\Delta P_{A, O_{j+1}} & \equiv P\left(x_{3}<O_{j+1}, x_{4}>O_{j+1}\right)-P\left(x_{3}<0, x_{4}>0\right) \\
\text { or } & \equiv P\left(x_{3}>O_{j+1}, x_{4}<O_{j+1}\right)-P\left(x_{3}>0, x_{4}<0\right) \\
& =\frac{1}{\pi} \sqrt{\frac{1-\rho_{3,4}}{1+\rho_{3,4}}} \times \frac{O_{j+1}^{2}}{\sigma_{j+1}^{2}} .
\end{aligned}
$$

Thus, the total probability variation due to the offsets is

$$
\begin{aligned}
\Delta P_{j, j+1}^{z} & =\Delta P_{A, O_{j}}+\Delta P_{A, O_{j+1}} \\
& =\frac{1}{\pi} \sqrt{\frac{1-\rho_{1,2}}{1+\rho_{1,2}}} \times \frac{O_{j}^{2}}{\sigma_{j}^{2}}+\frac{1}{\pi} \sqrt{\frac{1-\rho_{3,4}}{1+\rho_{3,4}}} \frac{O_{j+1}^{2}}{\sigma_{j+1}^{2}} .
\end{aligned}
$$

Comparing (44) with (40), the weak-correlation case shows larger probability variation than the strong-correlation case. Thus, we choose (40) as the upper bound for $\Delta P_{j, j+1}^{z}$.

\section{APPENDIX C}

\section{Timing Fluctuation Due to Skew CaLibRation}

Consider the $(j-1)$ th calibration channel in the CP of Fig. 10. It receives the $x_{j-1}[k]$ and $x_{j}[k]$ sequences and generates the $T_{j}[k]$ output to adjust $\tau_{j}$. Its ACC 1 accumulator takes $U[k]=$ $m[k]-z_{j-1}[k]$ and generates $R[k]$. Define $P_{U}[n]$ as the probability function of $U[k]=n$ and $P_{R}[n]$ as the probability function of $R[k]=n$, where $n$ is an integer. Since $z_{j}[k] \in\{0,1\}$ and $m[k] \in\{0,1\}, P_{U}[n]=0$ for $n>1$ or $n<-1$. $R[k]$ is generated by accumulating the $U[k]$ sequence. It is reset to 0 whenever $R[k] \geq+N_{C}$ or $R[k] \leq-N_{C}$. Thus, $P_{R}[n]=0$ for $n>+N_{C}$ or $n<-N_{C}$. We can derive the relationship between $P_{R}[n]$ and $P_{U}[n]$ as follows. At $k=1$, let $U[1]=R[1]=$ $n$, then the probability mass function (PMF) of $R[1]=n$ is the same as the PMF of $U[1]=n$. At $k=2$, the condition for $R[2]=1$ is $U[1]=1 \& U[2]=0$ or $U[1]=0 \& U[2]=1$. Thus, the probability of $R[2]=1$ is $P_{U}[+1] \times P_{U}[0]+P_{U}[0] \times$ $P_{U}[+1]=2 P_{U}[+1] \times P_{U}[0]$. From the same reasoning, the probability mass function of $R[2]=n$ is $P_{U}[n] * P_{U}[n]$, where

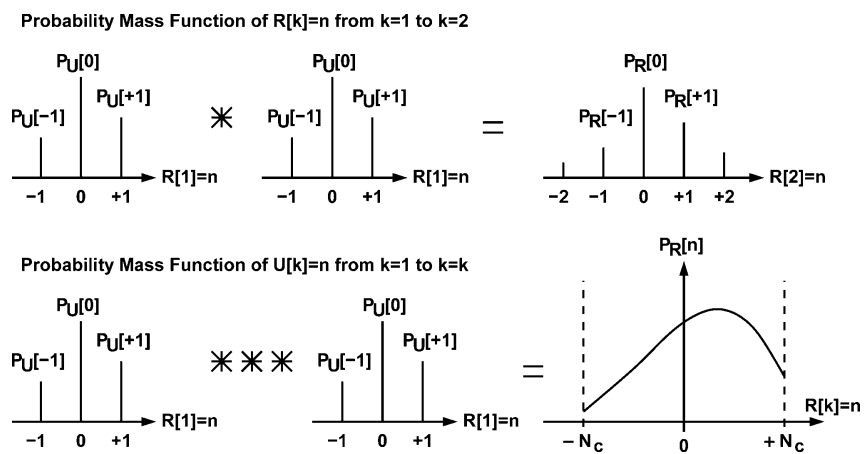

Fig. 21. Relationship between $P_{R}[n]$ and $P_{U}[n]$.

$*$ is a convolution operator. As shown in Fig. 21, we can also derive that $P_{R}[n]$ is a consecutive $k$ times convolution of $P_{U}[n]$, i.e.,

$$
P_{R}[n]=P_{U}[n] * P_{U}[n] * \cdots * P_{U}[n] .
$$

When the calibration loop converges, it implies $k \rightarrow \infty$ and $P_{R}[n] * P_{U}[n]=P_{R}[n]$. However, the BPD following the ACC1 accumulator reset $R[k]$ to zero whenever $R[k]=+N_{C}$ or $-N_{C}$. This means $P_{R}[n]=0$ for $n>+N_{C}$ or $n<-N_{C}$. The probability of $P_{R}\left[+N_{C}+1\right]=P_{R}\left[+N_{C}\right] \times P_{U}[+1]$ is reset to zero and added to $P_{R}[0]$. The probability of $P_{R}\left[-N_{C}-1\right]=$ $P_{R}\left[-N_{C}\right] \times P_{U}[-1]$ is also reset to zero and added to $P_{R}[0]$. We can find $P_{R}[n]$ by solving the following recursive difference equations:

$$
\begin{aligned}
P_{R}\left[+N_{C}\right]= & P_{R}\left[+N_{C}-1\right] P_{U}[+1] \\
P_{R}\left[-N_{C}\right]= & P_{R}\left[-N_{C}+1\right] P_{U}[-1] \\
P_{R}\left[+N_{C}-1\right]= & P_{R}\left[+N_{C}-2\right] P_{U}[+1] \\
& +P_{R}\left[+N_{C}-1\right] P_{U}[0] \\
P_{R}\left[-N_{C}+1\right]= & P_{R}\left[-N_{C}+2\right] P_{U}[-1] \\
& +P_{R}\left[-N_{C}+1\right] P_{U}[0] \\
P_{R}[n]= & P_{R}[n-1] P_{U}[+1]+P_{R}[n] P_{U}[0] \\
& +P_{R}[n+1] P_{U}[-1]
\end{aligned}
$$

where $-N_{C}+2 \leq n \leq N_{C}-2$.

The characteristic equation is

$$
y=P_{U}[+1]+P_{U}[0] y+P_{U}[-1] y^{2}
$$

where $y$ is the characteristic value for the above recursive equations. Solving the characteristic equation gives $y=1$ or

$$
y=\frac{P_{U}[+1]}{P_{U}[-1]} \equiv y_{r}
$$

With this and $P_{U}[0]+P_{U}[+1]+P_{U}[-1]=1$, the general form of $P_{R}[n]$ can be written as

$$
P_{R}[n]=A+B\left(y_{r}\right)^{n} .
$$

The above recursive difference equations can be summarized as

$$
P_{R}[n]=\frac{P_{R}\left[+N_{C}\right]}{P_{U}[+1]\left(y_{r}^{-1}-1\right)}\left(-1+y_{r}^{-N_{C}+n}\right)
$$




$$
\begin{aligned}
M\left(\tau_{j, 0}\right) \frac{\Delta P\left(\tau_{j, 0}\right)}{N_{C}-1} & =M\left(\tau_{j,-1}\right) \frac{\Delta P\left(\mu_{t}-\tau_{j, 0}\right)}{N_{C}-1} \\
M\left(\tau_{j, 0}\right) \frac{\Delta P\left(\tau_{j, 0}\right)}{N_{C}\left(N_{C}-1\right) \Delta y_{0}} & =M\left(\tau_{j,+1}\right) \frac{\Delta P\left(\mu_{t}+\tau_{j, 0}\right)}{N_{C}-1} \\
M\left(\tau_{j,-2}\right) \frac{\Delta P\left(2 \mu_{t}-\tau_{j, 0}\right)}{N_{C}-1} & =M\left(\tau_{j,-1}\right) \frac{\Delta P\left(\mu_{t}-\tau_{j, 0}\right)}{N_{C}\left(N_{C}-1\right)\left(\frac{\mu_{t}}{T_{s}-\Delta y_{0}}\right)} \\
M\left(\tau_{j, 1}\right) \frac{\Delta P\left(\mu_{t}+\tau_{j, 0}\right)}{N_{C}\left(N_{C}-1\right)\left(\frac{\mu_{t}}{T_{s}+\Delta y_{0}}\right)} & =M\left(\tau_{j, 2}\right) \frac{\Delta P\left(2 \mu_{t}+\tau_{j, 0}\right)}{N_{C}-1}
\end{aligned}
$$

$$
\frac{P_{R}\left[+N_{C}\right]}{P_{R}\left[-N_{C}\right]}=\frac{-1+y_{r}^{+N_{C}}}{+1-y_{r}^{-N_{C}}} \equiv \eta
$$

where $-N_{C}+2 \leq n \leq N_{C}-2$ and $y_{r} \neq 1 . P_{R}\left[+N_{C}\right]$ shows the probability of $\tau_{j}$ being increased and $P_{R}\left[-N_{C}\right]$ shows the probability of $\tau_{j}$ being decreased. The $y_{r}^{+N_{C}}$ and $y_{r}^{-N_{C}}$ terms in (55) imply that the $P_{R}\left[+N_{C}\right] / P_{R}\left[-N_{C}\right]$ ratio is either very large or close to 0 if $N_{C}$ is large. The above statement is still valid even when the difference between $P_{U}[+1]$ and $P_{U}[-1]$ is small.

When the timing-skew calibration loop converges, $y_{r}$ is close to 1 , Define $y_{r}=1+\Delta y$. From (55), we have

$$
\frac{P_{R}\left[+N_{C}\right]}{P_{R}\left[-N_{C}\right]} \approx \begin{cases}N_{C} \Delta y, & \text { if } \Delta y>0 \\ \frac{-1}{\left(N_{C} \Delta y\right)}, & \text { if } \Delta y<0\end{cases}
$$

Note that

$\lim _{\Delta y \rightarrow 0^{+}} \frac{P_{R}\left[+N_{C}\right]}{P_{R}\left[-N_{C}\right]} \neq \lim _{\Delta y \rightarrow 0^{-}} \frac{P_{R}\left[+N_{C}\right]}{P_{R}\left[-N_{C}\right]} \neq\left.\frac{P_{R}\left[+N_{C}\right]}{P_{R}\left[-N_{C}\right]}\right|_{\Delta y=0}$.

The case with $\Delta y>0$ is symmetical to the case with $\Delta y<0$. To ease discussion, only the $\Delta y>0$ case is considered below. We have

$$
\begin{aligned}
P_{R}\left[+N_{C}\right] & =\frac{\Delta P}{N_{C}-1} \\
P_{R}\left[-N_{C}\right] & =\frac{\Delta P}{N_{C}\left(N_{C}-1\right) \Delta y}
\end{aligned}
$$

where $\Delta P=P_{U}[+1]-P_{U}[-1]$ is a function of the $\tau_{j}$ timing skew.

Consider a $x(t)$ signal that has an uniform ZC density of $Z_{R}$. Let $\tau_{j, 0}=\Delta y_{0} T_{s}$. When the calibration loop converges, we have $P_{U}[+1]=Z_{R}\left(1+\Delta y_{0}\right)$ and $P_{U}[-1]=Z_{R}$. Furthermore, the probability of $\tau_{j}$ jumping from $\tau_{j, i}$ to $\tau_{j, i+1}$ is equal to the probability of $\tau_{j}$ jumping from $\tau_{j, i+1}$ to $\tau_{j, i}[12]$, where $i$ is an integer and $\tau_{j, i}$ is a discrete value of $\tau_{j}$. Therefore, see (60)-(63) at the top of the page. Note that $\sum_{i=-\infty}^{\infty} M\left(\tau_{j, i}\right)=1$. If $N_{C}$ is large, only $M\left(\tau_{j, 0}\right), M\left(\tau_{j,-1}\right), M\left(\tau_{j, 1}\right), M\left(\tau_{j,-2}\right)$ and $M\left(\tau_{j, 2}\right)$ are significant. We have

$$
M\left(\tau_{j,-1}\right)=\frac{\tau_{j, 0}}{\mu_{t}-\tau_{j, 0}} M\left(\tau_{j, 0}\right)
$$

$$
\begin{aligned}
M\left(\tau_{j, 1}\right) & =\frac{T_{s}}{N_{C}\left(\mu_{t}+\tau_{j, 0}\right)} M\left(\tau_{j, 0}\right) \\
M\left(\tau_{j,-2}\right) & =\frac{\Delta t \times T_{s}}{N_{C}\left(\mu_{t}-\tau_{j, 0}\right) \times\left(2 \mu_{t}-\tau_{j, 0}\right)} M\left(\tau_{j, 0}\right) \\
M\left(\tau_{j, 2}\right) & =\frac{T_{s}^{2}}{N_{C}^{2}\left(2 \mu_{t}+\tau_{j, 0}\right)\left(\mu_{t}+\tau_{j, 0}\right)} M\left(\tau_{j, 0}\right) .
\end{aligned}
$$

Then, $M\left(\tau_{j, i}\right)$ can be approximated by

$$
\begin{aligned}
M\left(\tau_{j, 0}\right) & =\frac{\mu_{t}-\tau_{j, 0}}{\mu_{t}} \\
M\left(\tau_{j,-1}\right) & =\frac{\tau_{j, 0}}{\mu_{t}} \\
M\left(\tau_{j, 1}\right) & =\frac{T_{s}}{N_{C} \mu_{t}\left(\mu_{t}+\tau_{j, 0}\right)}\left(\mu_{t}-\tau_{j, 0}\right) \\
M\left(\tau_{j,-2}\right) & =\frac{\tau_{j, 0} \times T_{s}}{N_{C} \mu_{t}\left(2 \mu_{t}-\tau_{j, 0}\right)} \\
M\left(\tau_{j, 2}\right) & =\frac{T_{s}^{2}}{N_{C}{ }^{2}\left(2 \mu_{t}+\tau_{j, 0}\right)\left(\mu_{t}+\tau_{j, 0}\right)} \frac{\mu_{t}-\tau_{j, 0}}{\mu_{t}} .
\end{aligned}
$$

The mean variance of $\tau_{j, i}$ is

$$
\begin{aligned}
\sigma^{2}(\tau) \equiv & \frac{1}{\mu_{t}} \int_{-\mu_{t} / 2}^{+\mu_{t} / 2} \sum_{i=-\infty}^{+\infty}\left[M\left(\tau_{j, i}\right) \times\left(\tau_{j, 0}+i \mu_{t}\right)^{2}\right] \\
& \times d \tau_{j, 0} \\
= & \frac{2}{\mu_{t}} \int_{0}^{+\mu_{t} / 2} \sum_{i=-\infty}^{+\infty}\left[M\left(\tau_{j, i}\right) \times\left(\tau_{j, 0}+i \mu_{t}\right)^{2}\right] \\
& \times d \tau_{j, 0} \\
\approx & \frac{1}{6} \mu_{t}^{2}+\frac{4 T_{s}}{3 N_{C}} \mu_{t} .
\end{aligned}
$$

The above equation is repeated in (14). We can ignore the case with $y_{r}=1$. In this case, $\tau_{j, 0}=0$, thus the integration in the above equation is zero, i.e., $\int_{0}^{0}$ (any function) $d \tau_{j, 0}=0$.

\section{REFERENCES}

[1] Y. Jenq, "Digital spectra of non-uniformly sampled signals: Theories and applications. III. Arobust sampling time offset estimation algorithm for ultra high speed waveform digitizers using interleaving," IEEE Trans. Instrum. Meas., vol. 39, no. 1, pp. 71-75, Feb. 1990. 
[2] J. Elbornsson, F. Gustafsson, and J.-E. Eklund, "Blind adaptive equalization of mismatch errors in a time-interleaved A/D converter system," IEEE Trans. Circuits Syst. I, Reg. Papers, vol. 51, no. 1, pp. 151-158, Jan. 2004.

[3] S. M. Jamal, D. Fu, N. C.-J. Chang, P. J. Hurst, and S. H. Lewis, “A 10-b 120-Msample/s time-interleaved analog-to-digital converter with digital background calibration," IEEE J. Solid-State Circuits, vol. 37 , no. 12, pp. 1618-1627, Dec. 2002.

[4] H. Jin and E. K. F. Lee, "A digital-background calibration technique for minimizing timing-error effects in time-interleaved ADC's," IEEE Trans. Circuits Syst. II, Analog Digit. Signal Process., vol. 47, no. 7, pp. 603-613, Jul. 2000.

[5] E. Iroaga and B. Murmann, "A background correction technique for timing errors in time-interleaved analog-to-digital converters," in Proc. IEEE Int. Symp. Circuits Syst., May 2005, pp. I-5557-I-5560.

[6] J. Christiansen, "An integrated high resolution CMOS timing generator based on an array of delay locked loops," IEEE J. Solid-State Circuits, vol. 31, no. 7, pp. 952-957, Jul. 1996.

[7] M. Mota and J. Christiansen, "A high-resolution time interpolator based on a delay-locked loop and $R C$ delay line," IEEE J. Solid-State Circuits, vol. 34, no. 10, pp. 1360-1366, Oct. 1999.

[8] F. Baronti, D. Lunardini, R. Roncella, and R. Saletti, "A self-calibrating delay-locked delay line with shunt-capacitor circuit scheme," IEEE J. Solid-State Circuits, vol. 39, no. 2, pp. 384-387, Feb. 2004.

[9] C.-Y. Wang and J.-T. Wu, "A background timing-skew calibration technique for time-interleaved analog-to-digital converters," IEEE Trans. Circuits Syst. II, Reg. Papers, vol. 53, no. 4, pp. 299-303, Apr. 2006.

[10] H. Stark and J. W. Woods, Probability Random Processes and Estimation Theory for Engineers, 2nd ed. Englewood Cliffs, NJ: Prentice-Hall, 1995.

[11] J. T. Barnett and B. Kedem, "Zero-crossing rates of functions of Gaussian processes," IEEE Trans. Inform. Theory, vol. 37, no. 4, pp. 1188-1194, Jul. 1991.

[12] C.-C. Huang and J.-T. Wu, "A background comparator calibration technique for flash analog-to-digital converters," IEEE Trans. Circuits Syst. I, Reg. Papers, vol. 52, no. 9, pp. 1732-1740, Sep. 2005.

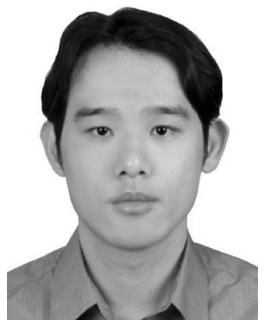

Chung-Yi Wang was born in Tai-Chung, Taiwan. He received the B.S. degree and the M.S. degree in electronics engineering from National Chiao-Tung University, Hsin-Chu, Taiwan, in 2002 and 2003, respectively, where he is currently working toward the Ph.D. degree.

His research interests are in mixed-signal, highspeed, and high-resolution integrated circuits design in data communication.

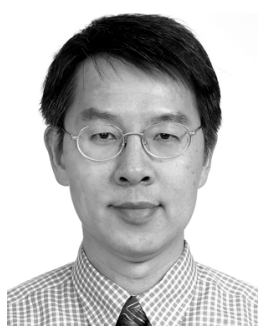

Jieh-Tsorng Wu (S'83-M'87-SM'06) was born in Taipei, Taiwan. He received the B.S. degree in electronics engineering from National Chiao-Tung University, Hsin-Chu, Taiwan, in 1980, and the M.S. and $\mathrm{Ph} . \mathrm{D}$. degrees in electrical engineering from Stanford University, Stanford, CA, in 1983 and 1988 , respectively.

From 1980 to 1982, he served in the Chinese Army as a Radar Technical Officer. From 1982 to 1988, at Stanford University, he focused his research on highspeed analog-to-digital conversion in CMOS VLSI.

From 1988 to 1992, he was a Member of Technical Staff with Hewlett-Packard Microwave Semiconductor Division, San Jose, CA, and was responsible for several linear and digital giga-hertz IC designs. Since 1992, he has been with the Department of Electronics Engineering, National Chiao-Tung University, where he is now a Professor. His current research interests are high-performance mixed-signal integrated circuits.

Dr. Wu is a member of Phi Tau Phi. 